\title{
Embryonic Heat Conditioning Induces TET-Dependent Cross-Tolerance to Hypothalamic Inflammation Later in Life
}

\author{
Tali Rosenberg ${ }^{1,2}$, Tatiana Kisliouk ${ }^{1}$, Tomer Cramer ${ }^{1,2}$, Dmitry Shinder ${ }^{1}$, Shelly Druyan ${ }^{1}$ \\ and Noam Meiri ${ }^{1 *}$
}

${ }^{1}$ Agricultural Research Organization, Volcani Center, Institute of Animal Science, Rishon LeZion, Israel, ${ }^{2}$ Department of Animal Science, The Robert H. Smith Faculty of Agriculture, Food and Environment, The Hebrew University of Jerusalem, Rehovot, Israel

Early life encounters with stress can lead to long-lasting beneficial alterations in the response to various stressors, known as cross-tolerance. Embryonic heat conditioning $(\mathrm{EHC})$ of chicks was previously shown to mediate resilience to heat stress later in life. Here we demonstrate that $\mathrm{EHC}$ can induce cross-tolerance with the immune system, attenuating hypothalamic inflammation. Inflammation in EHC chicks was manifested, following lipopolysaccharide (LPS) challenge on day 10 post-hatch, by reduced febrile response and reduced expression of LITAF and $N F_{K} B$ compared to controls, as well as nuclear localization and activation of $\mathrm{NF}_{\kappa} B$ in the hypothalamus. Since the crosstolerance effect was long-lasting, we assumed that epigenetic mechanisms are involved. We focused on the role of ten-eleven translocation (TET) family enzymes, which are the mediators of active CpG demethylation. Here, TET transcription during early life stress was found to be necessary for stress resilience later in life. The expression of the TET family enzymes in the midbrain during conditioning increased in parallel to an elevation in concentration of their cofactor $\alpha$-ketoglutarate. In-ovo inhibition of TET activity during $\mathrm{EHC}$, by the $\alpha$-ketoglutarate inhibitor bis-2-(5-phenylacetamido-1,3,4-thiadiazol-2-yl) ethyl sulfide (BPTES), resulted in reduced total and locus specific CpG demethylation in 10-day-old chicks and reversed both thermal and inflammatory resilience. In addition, EHC attenuated the elevation in expression of the stress markers HSP70, CRHR1, and $C R H R 2$, during heat challenge on day 10 post-hatch. This reduction in expression was reversed by BPTES. Similarly, the EHC-dependent reduction of inflammatory gene expression during LPS challenge was eliminated in BPTES-treated chicks. Thus, TET family enzymes and $\mathrm{CpG}$ demethylation are essential for the embryonic induction of stress cross-tolerance in the hypothalamus.

Keywords: embryonic heat conditioning, epigenetics, TET, hypothalamus, chicks

\section{INTRODUCTION}

All stressful exposures have an impact on the brain, regardless of whether they occur during the pre- or postnatal period, infancy or adulthood (Lupien et al., 2009). Emerging scientific consensus argues that the origins of adult disease, psychopathologies included, are often found among developmental and biological disruptions occurring during development and the early 
years of life. Either biological embedding of adversities during sensitive developmental periods, or accumulated damage gathered over time, can severely influence the formation of such diseases during adulthood (Shonkoff et al., 2009; Taylor, 2010; Labonté et al., 2012). These embryonic or early life environmental influences can lead to different stress responses, including vulnerability or resilience. Since these environmental effects are long-lasting, epigenetic regulatory mechanisms are probably involved and are key factors in the establishment and tuning of adult stress responses (Klengel and Binder, 2015; Turecki and Meaney, 2016; Horowitz, 2017; Cramer et al., 2018). The acquired stress response can have a broader impact than merely an adjusted response to the encountered stressor; it can also have a cross effect with other stress occurrences.

Cross-tolerance describes resilience to one stressor acquired by conditioning to a different stressor. For example, heat acclimation has been shown to improve cardiovascular function as well as protect against ischemia (Horowitz et al., 2015; Pollak et al., 2017). Here, we were interested in the cross-tolerance effect of embryonic heat conditioning (EHC) of chicks. The chick is an optimal model for studying manipulations during the embryonic period, because embryonic manipulations can be performed without affecting the maternal environment, and the chick is completely independent of maternal guidance after hatch (Haller et al., 2015; Goodfellow et al., 2016; Duman et al., 2018). EHC performed during the time of hypothalamic-pituitary-adrenal (HPA) axis development has been shown to improve thermal tolerance later in life (Piestun et al., 2009). Although the HPA axis has been shown to be involved in the development of stress, it has also been implicated in central nervous system (CNS) inflammation (Hueston and Deak, 2014). Here, EHC was used to induce cross-tolerance with hypothalamic inflammation later in life.

The hypothalamus is a major contributor to the development of stress responses (Smith and Vale, 2006), but it is also a key regulator of energy and thermal homeostasis (Morrison, 2016). Various studies have demonstrated that early life stress is a contributing factor to adult inflammation (Pace et al., 2006; Miller et al., 2009; Carpenter et al., 2010; Gouin et al., 2012; Fagundes et al., 2013). Hypothalamic inflammation is key during the onset of obesity (Roesslein et al., 2015; André et al., 2017; Jais and Brüning, 2017), and aggravates the neurological damage incurred from stroke, heat stroke, or traumatic brain injury (Arvin et al., 1996; Emsley et al., 2008; Macrez et al., 2011; Chao et al., 2015). In this work, we studied the effect of EHC on hallmarks of CNS inflammation in the hypothalamus.

CNS inflammation involves activation of TLR4/MyD88 nuclear factor kappa B (NFKB)-dependent transcription of inflammatory cytokines, such as tumor necrosis factor alpha (TNF $\alpha)$, interleukin (IL) $1 \beta$ and IL6, and an increase in nitric oxide (NO) production in the resident microglia (Wang et al., 2009; Tang and Le, 2016). TLR4 dependent NO production by resident microglia is triggered by nitric oxide synthase 2 (NOS2) which is expressed in neurons (Béchade et al., 2014). Moreover this inflammatory activation of NO leads to detrimental protein radical formation (Kumar et al., 2014). CNS inflammation is also characterized by blood brain barrier disruption derived by astrocyte production of vascular Endothelial Growth Factor A (VEGFA) (Argaw et al., 2012). Alternative, anti-inflammatory activation of microglia, characterized by the increased expression of cytokines IL4 and IL10, as well as mannose receptor C-type 1 (MRC1), and increased arginase activity, has been shown to be necessary for anti-inflammatory effects, as well as parasite clearance, allergic responses and angiogenesis (Martinez and Gordon, 2014). To study the effect of EHC on hypothalamic inflammation, we injected chicks with lipopolysaccharide (LPS) and measured the gene expression of the proinflammatory LPS-induced TNF factor (LITAF) and $N F \kappa B$, as well as the anti-inflammatory IL10 and MRC1. Previous study in laying hens, demonstrated that early life (5 days of age) heat stress had no effect on LPS reaction, later in life (Star et al., 2009), however very little is known regarding LPS reaction in the EHC model.

We focused on the epigenetic regulation during $\mathrm{EHC}$ that is implicated in creating cross-tolerance with reduced hypothalamic inflammation. Epigenetics is the study of factors, mostly environmental, that affect gene expression without changing the genetic sequence. There are different layers of epigenetic regulation, including DNA methylation inhibiting the association of enhancing or inhibitory elements, histone-tail modifications such as acetylation or methylation that change the gene's accessibility to trans elements, and microRNA regulation of transcription (Meaney and Szyf, 2005; Goldberg et al., 2007). CpG methylation is performed by DNA methyl transferases (DNMT's). This family of enzymes include DNMT1 that preserves DNA methylation during development (Hoffmann et al., 2015), DNMT3A and DNMT3B that perform de novo DNA methylation (Hoffmann et al., 2015). Recently, hydroxymethylation, leading to active $\mathrm{CpG}$ demethylation by the ten-eleven translocation (TET) family enzymes, was discovered (Kohli and Zhang, 2013). TET proteins have three main isoforms (TET1-3), all sharing a C-terminal catalytic domain with the ability to localize to the nucleus and perform $5 \mathrm{mC}$ oxidation. However, only full length TET1 and TET3 also contain an N-terminal CXXC, DNA binding domain. TET2 lost its CXXC binding domain due to genomic inversion during evolution, forming the adjacent gene IDAX, which enables TET2 DNA binding, but also mediates TET2 degradation. Moreover, TET proteins also display various catalytic independent regulation of gene transcription, mostly by association with histone modifiers ( $\mathrm{Wu}$ and Zhang, 2017; Melamed et al., 2018). TET1 expression is high in embryonic stem cells (ESC) and primordial germ cells (PGC), moreover, upon induction of pluripotency, both TET1 and TET2 expression is increased (Branco et al., 2012). TET2 mutations are abundant in hematopoietic malignancies, and cardiovascular diseases (Ferrone et al., 2020), as well as in increased inflammatory macrophage activation in TET2 knockouts (Fuster et al., 2017). TET3 expression peaks 
in oocytes and zygotes (Branco et al., 2012), and also in neurons (Wu and Zhang, 2017). Interestingly, DNMT3A, a de novo, DNA methyltransferase, inhibits TET2 and TET3 in hematopoietic malignancies (Gong et al., 2016). Epigenetic processes are influenced by the metabolic environment (Ruben et al., 2015) and different metabolites, such as acetyl$\mathrm{CoA}, \alpha$-ketoglutarate, NAD + and S-adenosylmethionine serve as regulators of epigenetic enzymes such as histone deacetylase, DNA methyltransferase and TET family enzymes (Baardman et al., 2015).

TET family enzymes have been reported to regulate different types of inflammation (Yang et al., 2016; Vento-Tormo et al., 2017; Hassan et al., 2018), as well as contribute to macrophage polarization and activation (Wallner et al., 2016). Although changes in DNA methylation during CNS inflammation have been previously reported (Ligthart et al., 2016), little is known about the role of TET family enzymes during CNS inflammation. Recently, the role of TET in the hypothalamus was explored during postnatal development in mice. Although TET expression was downregulated from birth to postnatal day 25, DNA hydroxymethylation increased (Cisternas et al., 2020), indicating a possible role during embryonic development of the hypothalamus. TET enzymes were also necessary to promote heat-stress resilience in chicks (Cramer et al., 2018, 2019). Here we demonstrate the role of TET family enzymes in the hypothalamus, during $\mathrm{EHC}$, in the establishment of cross-tolerance to hypothalamic inflammation later in life.

\section{MATERIALS AND METHODS}

\section{Experimental Model and Subject Details}

EHC was performed as previously described (Piestun et al., 2015). Briefly, we used 420 fertile Cobb strain broiler (Gallus domesticus) eggs from one breeder flock of hens. All eggs were obtained from Brown hatcheries (Hod Hasharon, Israel). The eggs were arbitrarily divided into two incubation treatment groups without application of specific randomization methods: control - eggs were incubated at $37.8^{\circ} \mathrm{C}$ and $56 \%$ relative humidity throughout the 21 days of the incubation period; EHC eggs were incubated at $39.5^{\circ} \mathrm{C}$ and $65 \%$ relative humidity for $12 \mathrm{~h} /$ day from embryonic day (ED) 7 to ED 16. The eggs were incubated in two type $65 \mathrm{Hs}$ automatic incubators (Masalles, Barcelona, Spain).

Hatched chicks were arbitrarily divided into pens, eight chicks per pen, in climate-controlled rooms at $32^{\circ} \mathrm{C}$ under a $22 / 2 \mathrm{~h}$ cycle of artificial illumination with ad libitum access to food and water. On day 7 post-hatch, the temperature was changed to $30^{\circ} \mathrm{C}$ and chicks were further divided into five chicks per pen. On day 10 post-hatch, the chicks were subjected to either heat or LPS challenge. A previous experiment did not show any differences related to the sexes, so we did not distinguish between sexes in this work.

Heat challenge was performed as previously described (Kisliouk et al., 2017). Briefly, on day 10 post-hatch, both control and EHC experimental chick groups were thermally challenged by exposure to $36^{\circ} \mathrm{C}$ for $24 \mathrm{~h}$. The chicks' baseline body temperature was measured and they were sacrificed by decapitation $0,2,6$, and $24 \mathrm{~h}$ into the heat challenge.

For the LPS challenge, $0.3 \mu \mathrm{g}$ LPS (Sigma Aldrich, Rehovot, Israel) originated from Salmonella and its vehicle solution $(0.9 \% \mathrm{w} / \mathrm{v} \mathrm{NaCl}$, designated as saline), were administered by intracerebroventricular (ICV) injection into both control and EHC experimental chick groups. Since the brain has no pain receptors, and we wanted to minimize animal stress and suffering, no anesthetics were used for the ICV injections. The chicks' body temperature was measured and they were sacrificed by decapitation 0 and $6 \mathrm{~h}$ into the LPS challenge.

All challenges were initiated at 07:00 $\mathrm{h}$ and continued for 24 or $6 \mathrm{~h}$. Temperature measurements during the challenges were conducted blindly. To minimize stress and suffering, animals were killed by fast decapitation.

\section{Embryonic Inhibition of Glutaminase}

Chorioallantoic membrane of ED 12 embryos was injected with the glutaminase inhibitor bis-2-(5-phenylacetamido1,3,4-thiadiazol-2-yl) ethyl sulfide (BPTES) (Tocris, Bristol, United Kingdom) dissolved in DMSO and further diluted in $0.9 \%(\mathrm{w} / \mathrm{v}) \mathrm{NaCl}$ to a final concentration of $0.01 \%(\mathrm{v} / \mathrm{v})$ DMSO. Each embryo received $12.5 \mathrm{mg} / \mathrm{kg}$ BPTES-0.01\% DMSO dissolved in $0.9 \% \mathrm{NaCl}$ (designated BPTES) or $0.01 \%$ DMSO dissolved in $0.9 \% \mathrm{NaCl}$ (designated saline). Immediately following injection, eggs were sealed with hot glue and returned to their incubators.

\section{Tissue Collection}

For total RNA and DNA isolation, the brain area matching the anterior hypothalamus was dissected and immersed in RNALater pH 5.2 (5.3 M ammonium sulfate, $25 \mathrm{mM}$ sodium citrate, $20 \mathrm{mM}$ EDTA). For western blots, $\alpha$-ketoglutarate quantification, TET activity assay and global $5 \mathrm{mC}$ and $5 \mathrm{hmC}$, isolated tissues were immediately frozen in liquid nitrogen and stored at $-80^{\circ} \mathrm{C}$.

\section{Total RNA Isolation and Quantitative PCR}

Total RNA was isolated using TRI Reagent (Molecular Research Center, Cincinnati, OH, United States) according to the manufacturer's instructions. Hypothalamic RNA (0.5 $\mu \mathrm{g})$ was reverse-transcribed to single-stranded cDNA by Super Script II Reverse Transcriptase and oligo (dT) plus random primers (Thermo Fisher Scientific, Waltham, MA, United States). Quantitative PCR was performed with $10 \mathrm{ng}$ cDNA in a StepOnePlus Real Time PCR System (Applied Biosystems) with PerfeCta SYBR Green FastMix, ROX (Quanta BioSciences, Gaithersburg, MD, United States). Dissociation curves were analyzed following each real-time PCR to confirm the presence of only one product and the absence of primer dimer formation. The threshold cycle number $(\mathrm{Ct})$ for each tested gene $(\mathrm{X})$ was used to quantify the relative abundance of that gene using the formula

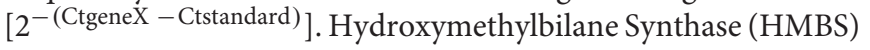
was used as the standard for mRNA expression. The primers used for real-time PCR were as follows $\left(5^{\prime} \rightarrow 3^{\prime}\right)$ : 


\begin{tabular}{|c|c|c|}
\hline Gene name & Primer FW & Primer REV \\
\hline HSP70 (Kisliouk et al., 2017) & TGGGTGTCTTCCAGCATGG & GATGAGGCGCTCTGTATCGG \\
\hline GILZ & CATGGAGGTGGCTGTCTATCAG & GGCACTGTTGTCCAGCTTCA \\
\hline EIF2B5 (Kisliouk et al., 2009) & GAAATCCAAGTGGTGCCG & GCATCAACATCTCGCAGCA \\
\hline CRHR1 (Kadhim et al., 2019) & CCCTGCCCCGAGTATT TCTA & СТT GСTCСTCTTCTССTCACTG \\
\hline LITAF & CCAGATGGGAAGGGAATGAA & CTACGGGTTGCTGCACATACA \\
\hline NFkB (Tan et al., 2014) & GTGTGAAGAAACGGGAACTG & GGCACGGTTGTCATAGATGG \\
\hline NOS2 & GATTGGGTGTGGATTGTACC & TGCATCCACCTGGTAGTAAA \\
\hline VEGFA & ACAAGAAAATCACTGTGAGCC & ACGTGAGTCTGTGAATTGC \\
\hline $1 L 10$ & ТТААСССАСТGССТСТСАСС & ССТTCTCGAACGTCTCCTTG \\
\hline DNMT1 (Cramer et al., 2019) & CCCGACC CCTATCGTGTG & AACTTCCAGATGCGCAG TाTG \\
\hline DNMT3A (Cramer et al., 2019) & AGTACCTCTCCATCTCCGTGCA & САСТСGСТССААСТСCATCAА \\
\hline DNMT3B (Cramer et al., 2019) & CCTGGAGTGTAACCCC GTTATG & CAAAGATCCTGTTCATCCCTGG \\
\hline
\end{tabular}

\section{Preparation of Cytosolic and Nuclear Fractions and Western Blotting}

Frozen anterior hypothalamus was lysed in PBS containing protease and phosphatase inhibitors (Sigma). Lysates were centrifuged at $1000 \mathrm{~g}$ for $8 \mathrm{~min}$ at $4^{\circ} \mathrm{C}$. The supernatant was added to Laemmli sample buffer and marked as the cytosolic fraction. The pellet was further lysed in nuclear extraction buffer $\left(20 \mathrm{mM}\right.$ Tris $\mathrm{pH} 7.8,125 \mathrm{mM} \mathrm{NaCl}, 5 \mathrm{mM} \mathrm{MgCl}_{2}$, $0.2 \mathrm{mM}$ EDTA, $12 \%$ glycerol, $0.1 \% \mathrm{NP}-40$ and protease and phosphatase inhibitors), sonicated and centrifuged at 1000 $g$ for $8 \mathrm{~min}$ at $4^{\circ} \mathrm{C}$. The supernatant was added to Laemmli sample buffer and marked as the nuclear fraction. Cytosolic and nuclear fractions were loaded onto a polyacrylamide gel for electrophoresis, then transferred to a nitrocellulose membrane and blocked with $3 \%$ skim milk (Sigma) for $1 \mathrm{~h}$ at room temperature. Membranes were incubated overnight with primary antibodies against NFKB (1:500, Abcam, Cambridge, United Kingdom, PRID: AB_443394), IKK $\alpha / \beta$ (1:500; Biorbit, Cambridge, United Kingdom, PRID: AB_2783519), p-Ser 176/177-IKK $\alpha+\beta$ (1:500, Life Span Biotechnology, Mandideep, Bhopal, India, PRID: AB_2783518), actin (1:6000; Cell Signaling Technology, Beverly, MA, United States, PRID: AB_330288) and H3 (1:2000, Cell Signaling Technology, PRID: AB_1904005), followed by three washes and incubation with secondary anti-rabbit IgG horseradish peroxidase-conjugated antibody (1:5000; Amersham Biosciences, Little Chalfont, United Kingdom, PRID: AB_772206) for $1 \mathrm{~h}$ at room temperature; after an additional three washes, membranes were reacted with SuperSignal Chemiluminescent substrates according to the manufacturer's instructions (Pierce Biotechnology, Rockford, IL, United States). Blots were imaged by the G:BOX chemi XRQ gel-imaging system (Syngene - Synoptics Ltd., Cambridge,
United Kingdom), and densitometric analysis was performed using Quantity One 1-D analysis software (Bio-Rad, Hercules, CA, United States).

\section{$\alpha$-Ketoglutarate Quantification}

All of the samples were analyzed for $\alpha$-ketoglutarate quantification in the same experiment, using Alpha Ketoglutarate (alpha KG) Assay Kit according to the manufacturer's protocols (Abcam).

\section{TET Activity}

TET activity was measured in the midbrain of chick embryos on ED 14 using the Epigenase 5mC-Hydroxylase TET Activity/Inhibition Assay Kit according to the manufacturer's protocols (EpiGentek, Farmingdale, NY, United States).

\section{DNA Methylation and Hydroxymethylation Analyses}

Total DNA methylation $(\% 5 \mathrm{mC})$ and hydroxymethylation (\%5hmC) were assessed using "MethylFlash" global DNA methylation ELISA kit and "MethylFlash" global DNA hydroxymethylation ELISA kit, accordingly (EpiGentek).

Sequence specific DNA methylation (\%5mC) and hydroxymethylation (\%5hmC) was assessed using the "EpiMark 5-hmC and 5-mC Analysis kit" (NEB, Ipswich, MA) according to the manufacturer's protocol. Briefly, DNA from day 10 post-hatch anterior hypothalamus, was incubated with T4 $\beta$-glucosyltransferase (T4-BGT, which adds a glucose mark on $5 \mathrm{hmC}$, creating $5 \mathrm{ghmC}), 37^{\circ} \mathrm{C}$, overnight. $\mathrm{T}^{-}-\mathrm{BGT}^{+}$ and $\mathrm{T}_{4}-\mathrm{BGT}^{-}$samples were than incubated with $M s p \mathrm{I}$ (cleaves DNA at CCGG, unless the middle CG is 5ghmC) or HpaII (cleaves DNA at CCGG unless the middle CG is modified to $5 \mathrm{mC}$, $5 \mathrm{hmC}$, or $5 \mathrm{ghmC}$ ) restriction enzymes, $37^{\circ} \mathrm{C}, 12 \mathrm{~h}$. The restriction products are later incubated with proteinase $\mathrm{K}, 40^{\circ} \mathrm{C}, 30 \mathrm{~min}$, followed by $10 \mathrm{~min}$ of $95^{\circ} \mathrm{C}$ for 
proteinase $\mathrm{K}$ inactivation. DNA samples were used in $\mathrm{qPCR}$ to determine $\% 5 \mathrm{hmC}$ and $\% 5 \mathrm{mC}$ according to the expression of specific targets.

$$
\% 5 h m C=\frac{\left(t_{5}-t_{1} \times\left(t_{6}: t_{3}\right)\right)}{t_{6}} \times 100, \% 5 m C=\frac{\left(t_{1} \times\left(t_{6}: t_{3}\right)\right)}{t_{6}} \times 100
$$

$\mathrm{t} 1=$ expression of $\mathrm{T} 4-\mathrm{BGT}^{+}, \mathrm{Msp}^{+} ; \mathrm{t} 3$ = expression of $\mathrm{T} 4-\mathrm{BGT}^{+}$, uncut; $\mathrm{t} 4=$ expression of $\mathrm{T}_{4}-\mathrm{BGT}^{-}, \mathrm{Msp}^{+} ; \mathrm{t} 5=$ expression of $\mathrm{T}_{4}-\mathrm{BGT}^{-}, \mathrm{Hpa}^{+}$; $\mathrm{t} 6=$ expression of $\mathrm{T} 4-\mathrm{BGT}^{-}$, uncut.

For qPCR we designed primers amplifying regions containing CCGG, which presented increased probability for binding transcription factors via the TFBIND algorithm ${ }^{1}$.

\begin{tabular}{lll}
\hline Gene name & Primer FW & Primer REV \\
\hline LITAF & AGCGAGTGCCGGTGG & CGACCCGGAGCAGTGAG \\
NFKB & GAAGCGCCGCCGGTT & CGACCTTCCTCCGGGCA \\
GILZ & ACAAGGTCACCCGGCT & ATAGGGACGCCGGGG \\
\hline
\end{tabular}

\section{Quantification and Statistical Analysis}

Statistical analysis was performed using GraphPad Prism 6 software (GraphPad Software, San Diego, CA, United States). All data were examined for normality by goodness of fit test and by Bartlett test for variance equality. As the distribution was normal, the parameters were not transformed. There was no sample size calculation. Nevertheless, the sample size was determined on the basis of previous studies (Kisliouk et al., 2017; Cramer et al., 2018). Sample number (n) included the number of individual chicks in each treatment group (indicated in Figure Legends). Means of two groups were compared by two-tailed unpaired $t$-test. Comparisons of LPS and conditioning effects were analyzed by two-way ANOVA followed by Sidak's or LSD multiple-comparison tests. Figure data are presented as mean \pm SEM, with exact $\mathrm{n}$ and $P$-values reported in the legends. The "Results" section provides the $F$ and $t$-test expressions.

\section{RESULTS}

\section{Cross-Tolerance: EHC Attenuates the Inflammatory Response in the Hypothalamus}

Chicks were heat-conditioned between ED 7 and 16. After hatch, chicks were raised in their optimal environmental temperature of $30^{\circ} \mathrm{C}$. Ten days after hatch, both $\mathrm{EHC}$ and control (nonconditioned) chicks were subjected to heat $\left(36^{\circ} \mathrm{C}\right)$ or LPS $(0.3 \mu \mathrm{g})$ challenge (Figure 1A). As a result of EHC, the body temperature of the EHC group on day 10 post-hatch was significantly lower than that of the control group $\left(t_{47}=2.03, P=0.047\right)$. The EHC group's body temperature remained significantly lower than that of the control group 2 and $6 \mathrm{~h}$ into the heat challenge $\left(t_{52}=2.28, P=0.026 ; t_{51}=3.27, P=0.002\right.$, respectively $)$ (Figure 1B). LPS challenge was performed on day 10 posthatch by ICV injection of either LPS or saline. As expected,

${ }^{1}$ http://tfbind.hgc.jp/
LPS induced a febrile response in both groups (LPS effect $F_{1}$, $108=64.16, P<0.0001)$, but the body temperature of the EHC-LPS group was significantly lower than that of the controlLPS group $6 \mathrm{~h}$ into the challenge $(P=0.005$; Figure 1C), indicating that the embryonic conditioning attenuated the febrile response during the LPS challenge (conditioning effect $F_{1,108}=12.59, P=0.0006$ ).

To determine whether the reduced febrile response to LPS is accompanied by reduced inflammation, we analyzed the expression patterns of both pro- and anti-inflammatory genes from the hypothalamic tissue of chicks sacrificed $6 \mathrm{~h}$ into the LPS challenge (Figure 1D). Following LPS challenge, the expression of the proinflammatory genes LITAF and NFKB was significantly reduced in the EHC group compared to controls (LITAF by $38 \%, t_{43}=2.63, P=0.01 ; N F \kappa B$ by $39 \%, t_{43}=3.91$, $P=0.0003)$. Furthermore, there was a significant increase in the expression of the anti-inflammatory genes IL10 and MRC1 (IL10 by $56 \%, t_{39}=3.28, P=0.002 ; M R C 1$ by $57 \%, t_{30}=2.57$, $P=0.03$; Figure 1D). Translocation of NFKB protein to the nucleus was also measured $6 \mathrm{~h}$ after LPS injection. Its cytosolic levels were increased in the EHC group $\left(t_{6}=2.83, P=0.03\right)$, and its nuclear levels were significantly reduced $\left(t_{6}=3.11\right.$, $P=0.02)$. Furthermore, IKK $\alpha / \beta$, which phosphorylates the regulatory subunit of $\mathrm{NF} \kappa \mathrm{B}$ to enable its nuclear translocation, and phosphorylated on its own Ser176/177, indicating IKK $\alpha / \beta$ activity (Gilmore, 2006), was less evident in the EHC group $\left(t_{6}=6.07, P=0.0009\right.$; Figure 1E).

\section{TET Family Enzymes Present Increased Expression During EHC}

Since we showed that EHC has a long-term effect on the expression of inflammatory genes, we assumed that epigenetic mechanisms are involved. Therefore, we measured mRNA expression of both the TET and the DNMT family enzymes in the midbrain during EHC on ED 10 and 14, and after the end of conditioning on ED 18 (Figure 2A). TET1 expression did not differ between EHC and control groups on ED $10\left(t_{18}=1.4\right.$, $P=0.18), 14\left(t_{15}=1.9, P=0.08\right)$, or $18\left(t_{18}=0.39, P=0.7\right)$. However, during conditioning, average TET1 expression in the EHC group increased from ED 10 to ED 14 (by 36\%), while control values remained constant. Moreover, TET1 expression on $\mathrm{ED} 18$, after conditioning, was lower in both control (by $34 \%$ compared to ED 10) and EHC (by $17 \%$ compared to ED 10; Figure 2B) groups. On ED 10, TET2 expression in the EHC group was significantly lower than in the control group $\left(t_{18}=3.1, P=0.006\right)$. However, on ED 14, TET2 expression was higher in the EHC group compared to the control group $\left(t_{15}=2.5, P=0.03\right)$. There was no difference in TET2 expression after the conditioning, on ED 18, between EHC and control groups $\left(t_{18}=0.34, P=0.74\right)$. Similar to TET1 expression, TET2 expression in the EHC group presented an increase between ED 10 and ED 14 (by 127\%), while the control, non-conditioned group displayed similar expression between ED 10 and ED 14. After the conditioning period on ED 18, TET2 expression returned to its level on ED 10 in the EHC group, and was lower for the control group (43\%; Figure 2C). TET3 expression did not differ between EHC and control groups on any of 
A

B
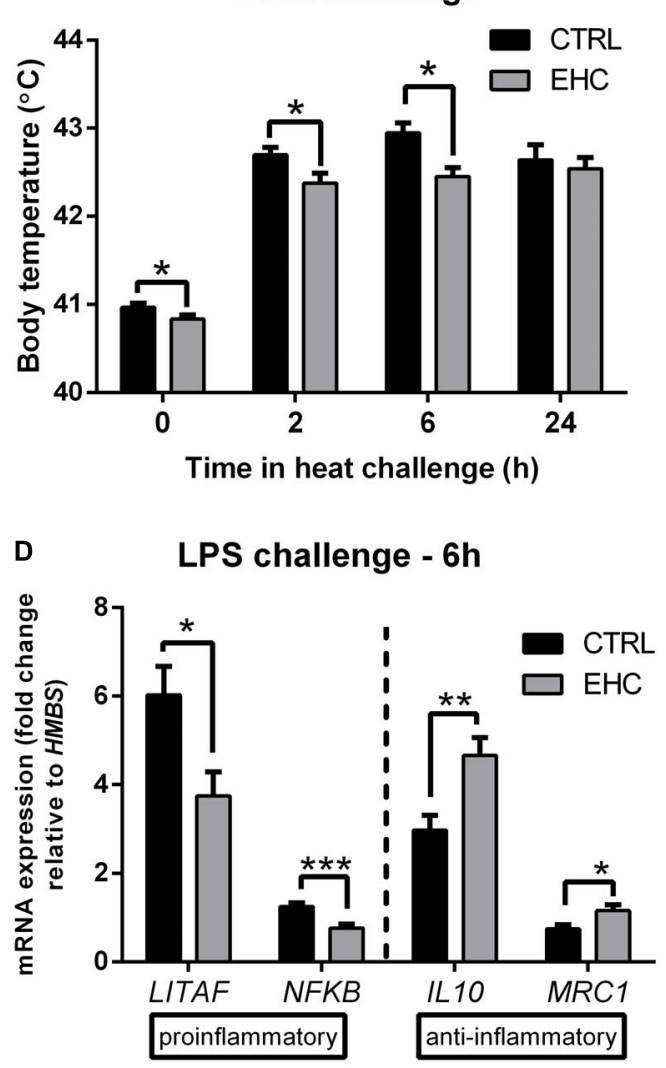

Embryonic period

ED 0-21

Conditioning

EHC

CTRL

$37.8^{\circ} \mathrm{C}, 56 \% \mathrm{RH}$
Day 10

posthatch

Challenge

$16 \quad 21$

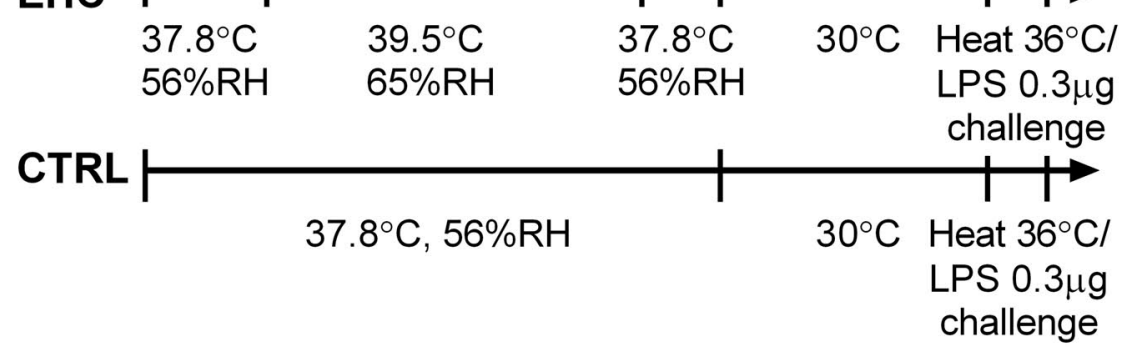

C LPS challenge - $6 \mathrm{~h}$

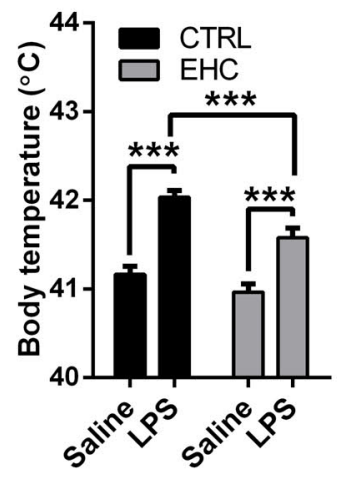

E

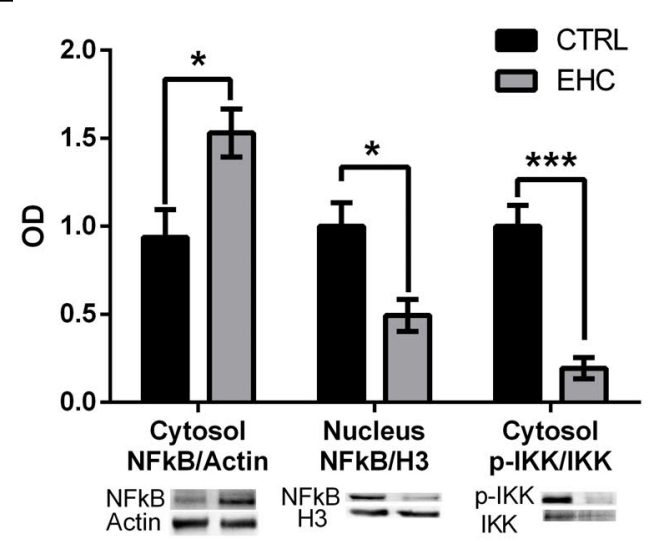

FIGURE 1 | Cross-tolerance: embryonic heat conditioning (EHC) attenuates the inflammatory response in the hypothalamus. (A) Schematic representation of the experiment. EHC on embryonic days (ED) 7-16, followed by heat/LPS challenge on day 10 post-hatch. (B) Body temperature measured 10 days post-hatch and at different times $(0,2,6,24 \mathrm{~h})$ into the heat challenge $\left(36^{\circ} \mathrm{C}\right) . \mathrm{t}_{0}\left(\mathrm{n}_{C T R L}=23, \mathrm{n}_{E H C}=27,{ }^{*} P=0.047\right) ; 2 \mathrm{~h}\left(\mathrm{n}_{C T R L}=26, \mathrm{n}_{E H C}=27,{ }^{*} P=0.026\right) ; 6 \mathrm{~h}\left(\mathrm{n}_{C T R L}=25\right.$, $\left.\mathrm{n}_{E H C}=27,{ }^{*} P=0.002\right) ; 24 \mathrm{~h}\left(\mathrm{n}_{C T R L}=17, \mathrm{n}_{E H C}=17, P=0.64\right)$. (C) Chicks (10 days old) were ICV-injected with LPS $(0.3 \mu \mathrm{g})$ or saline vehicle and their body temperature was measured $6 \mathrm{~h}$ after the injection. CTRL $\left(\mathrm{n}_{\text {saline }}=30, \mathrm{n}_{\text {LPS }}=29,{ }^{\star \star \star} P<0.0001\right)$; EHC $\left(\mathrm{n}_{\text {saline }}=27, \mathrm{n}_{L P S}=26,{ }^{\star \star \star} P<0.0001\right)$; LPS $\left(\mathrm{n}_{C T R L}=29\right.$, $\left.\mathrm{n}_{E H C}=26,{ }^{* *} \mathrm{P}=0.005\right)$. (D) Hypothalamic mRNA expression $6 \mathrm{~h}$ after LPS or saline injection; fold change of LITAF, NFKB, IL10, and MRC1 was normalized to HMBS, and reported relative to their respective saline controls. LITAF ( $\left.\mathrm{n}_{C T R L}=25, \mathrm{n}_{E H C}=20,{ }^{\star} P=0.012\right) ; N F_{K} B\left(\mathrm{n}_{C T R L}=25, \mathrm{n}_{E H C}=20,{ }^{\star \star \star} P=0.0003\right) ; / L 10$ $\left(\mathrm{n}_{C T R L}=22, \mathrm{n}_{E H C}=19,{ }^{*} P=0.002\right) ; M R C 1$ ( $\mathrm{n}_{C T R L}=6, \mathrm{n}_{E H C}=6,{ }^{*} P=0.03$ ). (E) Western blotting of cytosolic and nuclear fractions against NFKB, and cytosolic $I K K \alpha / \beta$ and $p-I K K \alpha / \beta, 6$ into LPS challenge. Cytosolic fraction proteins were normalized to actin and nuclear fraction proteins were normalized to histone $3(\mathrm{H} 3)$. Bottom panel shows the representative blots; top panel presents western quantification. Nuclear localization of NFKB/H3 ( $\left.\mathrm{n}_{C T R L}=4, \mathrm{n}_{E H C}=4,{ }^{*} P=0.02\right)$, cytosolic localization in the EHC group $\left({ }^{*} P=0.03\right)$, cytosolic IKK $\alpha / \beta$ phosphorylation $\left({ }^{* * *} P=0.0009\right)$. Data are presented as mean \pm SEM, and significant effects between groups are indicated as ${ }^{\star} 0.05<P<0.01,{ }^{\star \star} 0.01<P<0.001,{ }^{* \star} P<0.001$ using ANOVA test with LSD for multiple comparisons. CTRL, Control; RH, relative humidity. 
the experimental days. During the conditioning period, both groups presented increased TET3 expression between ED 10 and ED 14 (EHC by $200 \%$ and control by 100\%), and TET3 expression on ED 18, after the conditioning period, was reduced in both control (40\%) and EHC (48\%) groups relative to its level on ED 10 (Figure 2D). Overall, TET enzymes presented dynamic expression during the conditioning period, which may indicate their role during thermal conditioning and hypothalamic development. DNMTs expression did not differ between CTRL (non-conditioned) and EHC groups on all days of measurement except for DNMT3A on ED 10. DNMT1 expression increased between ED 10 and ED 14, and returned to baseline expression on $\mathrm{ED} 18\left(\mathrm{ED} 10: t_{18}=1.90 ; P=0.07\right.$; ED 14: $t_{15}=0.58 ; P=0.57$; ED 18: $t_{18}=1.02, P=0.32$; Figure 2E). DNMT3A expression was significantly lower in the EHC group on ED $10\left(t_{18}=2.49\right.$; $P=0.02)$ and its expression on ED 14 tended to increase compared to CTRL group $\left(t_{15}=1.81 ; P=0.09\right)$. There was no difference in DNMT3A expression during ED $18\left(t_{18}=0.24\right.$, $P=0.81$; Figure 2F). DNMT3B expression did not differ between CTRL and EHC groups on all days of measurement (ED 10: $t_{18}=1.86 ; P=0.08$; ED 14: $t_{15}=0.64 ; P=0.53$; ED 18: $t_{18}=0.38$, $P=0.71 ;$ Figure 2G).

\section{TET Enzyme Cofactor $\alpha$-Ketoglutarate Concentration in the Midbrain Is Increased and Necessary for TET Activity During EHC}

Since we found TET expression to be dynamic in the midbrain during EHC, we measured the concentration of its cofactor $\alpha$ ketoglutarate (Figure 3A), which has been found to metabolically and epigenetically regulate macrophage inflammatory activation (Liu et al., 2017). The EHC group presented increased levels of $\alpha$ ketoglutarate during conditioning $\left(\mathrm{ED} \mathrm{10:} \mathrm{t}_{6}=3.12, P=0.02\right.$; ED 14: $t_{14}=3.76, P=0.002$ ), but not after the conditioning period (ED 18: $t_{12}=0.76, P=0.46$; Figure 3B). Next, we inhibited $\alpha$ ketoglutarate accumulation during EHC by intra-ovo injection of BPTES, a glutaminase inhibitor, or saline into the blood vessels of the chorioallantoic membrane of ED 12 embryos. The embryos were returned to the incubator immediately after injection and continued their development until ED 14 and ED 18 (Figure 3C). To check the effectiveness of the inhibition, we first measured the concentration of $\alpha$-ketoglutarate during conditioning in the embryo midbrains on ED 14, and after conditioning on ED 18 (Figure 3D). BPTES reduced the amount of midbrain $\alpha$ ketoglutarate on both ED $14\left(t_{14}=5.05, P=0.0002\right)$ and ED $18\left(t_{14}=2.69, P=0.02\right.$; Figure $\left.3 \mathrm{D}\right)$. TET family enzyme activity was also measured on ED 14 in saline- and BPTES-injected, nonconditioned and EHC chicks (Figure 3E). Multiple comparisons demonstrated a significant increase in TET activity in the salineinjected EHC group compared to the saline-injected controls $(P<0.0001)$, whereas there was no difference between BPTESinjected EHC and control groups $(P=0.99)$. There was also a strong interaction $\left(F_{2}, 23=7.796, P=0.003\right)$ between the BPTES effect $\left(F_{2}, 23=29.34, P<0.0001\right)$ and the conditioning effect $\left(F_{1}\right.$, $23=21.45, P=0.0001 ;$ Figure $3 E$ ). These results led us to conclude that $\alpha$-ketoglutarate is necessary for TET enzyme activity, which is increased during EHC.

\section{TET Inhibition During EHC Has a Long-Lasting Effect, Blocking Heat Resilience Later in Life}

After establishing that TET is inhibited in the embryo by BPTES injection, we aimed to assess the long-term effect of embryonic TET inhibition (Figure 4A). Since the substrate for the TET family enzymes is $5 \mathrm{mC}$, and a major part of its product is $5 \mathrm{hmC}$, we measured the total $\% 5 \mathrm{mC}$ and $\% 5 \mathrm{hmC}$ on day 10 post-hatch, without heat/LPS challenge (Figures 4B,C). The EHC-BPTES group presented increased $\% 5 \mathrm{mC}$ compared to the $\mathrm{EHC}-$ saline group $(P=0.02)$, but there was no difference in $\% 5 \mathrm{mC}$ between the saline-injected control and EHC groups $(P=0.42$; Figure 4B), indicating that BPTES impaired TET activity. The total $5 \mathrm{hmC} \%$ was increased in the saline-injected EHC group compared to the saline-injected control group $(P=0.002)$. This increase in $\% 5 \mathrm{hmC}$ was blocked by BPTES, as the BPTES-injected EHC group did not differ from the saline-injected control group $(P=0.96)$, and $\% 5 \mathrm{hmC}$ was significantly lower than in the saline-injected EHC group $(P=0.006$; Figure $4 \mathrm{C})$. These results indicated that EHC activation of TET has a long-term effect, past the embryonic period.

Our next aim was to assess whether these epigenetic changes in TET activity during EHC underlie the heat resilience later in life. However, since BPTES inhibits glutaminolysis, and glutamate receptors in the preoptic hypothalamus play a role in thermal regulation (Sengupta et al., 2016), we wanted to determine whether BPTES affected other aspects of development. Therefore, we measured the chick's body weight at hatch, and found no difference between naïve (non-injected), salineinjected or BPTES-injected chicks $\left(F_{2}, 182=1.216, P=0.299\right.$; Figure 4D). Next, we introduced 10-day-old chicks which had been previously injected with BPTES or saline during embryogenesis to heat challenge, and measured their body temperature before $\left(\mathrm{t}_{0} ;\right.$ Figure $\left.4 \mathrm{E}\right)$ and $6 \mathrm{~h}$ into the heat challenge (Figure 4F). BPTES did not have any effect on baseline body temperature $\left(F_{2}, 34=0.365, P=0.697\right.$; Figure $\left.4 \mathrm{E}\right)$, further indicating that glutamatergic inhibition on ED 14 does not affect thermal regulation later in life. During heat challenge, there was a significant decrease in the saline-injected EHC group's body temperature compared to the saline-injected control group $(P=0.004$; Figure $4 \mathbf{F})$, indicating thermal resilience, supported by a strong conditioning effect $\left(F_{1}, 24=5.683\right.$, $P=0.03)$. The BPTES-injected EHC group presented higher body temperature than the saline-injected EHC group $(P=0.02)$, and no difference compared to the BPTES-injected control group $(P=0.99)$, indicating that BPTES blocked thermal resilience (Figure 4F). These findings are supported by the significant interaction $\left(F_{1}, 24=8.371, P=0.008\right)$ between BPTES and conditioning. It is worth noting that there was no difference between the BPTES-injected control and BPTES-injected EHC group body temperatures $(P=0.93)$ during the heat challenge, indicating that glutamatergic transmission affecting thermal regulation was not damaged by the in-ovo injection of BPTES 

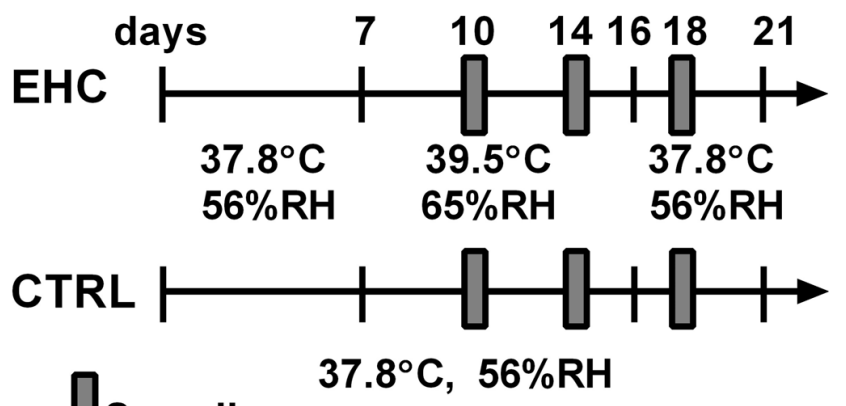

B

TET1

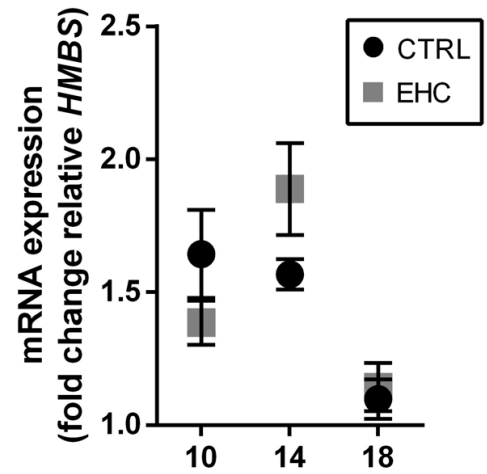

Embryonic day

E

DNMT1

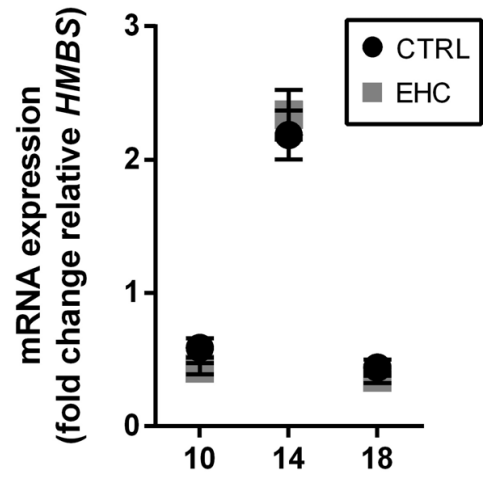

Embryonic day
TET2

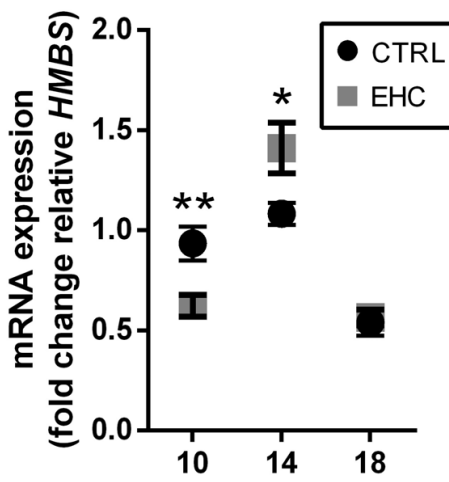

Embryonic day

$\mathbf{F}$

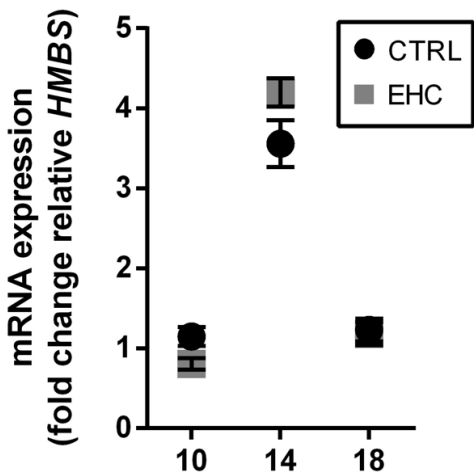

Embryonic day
D

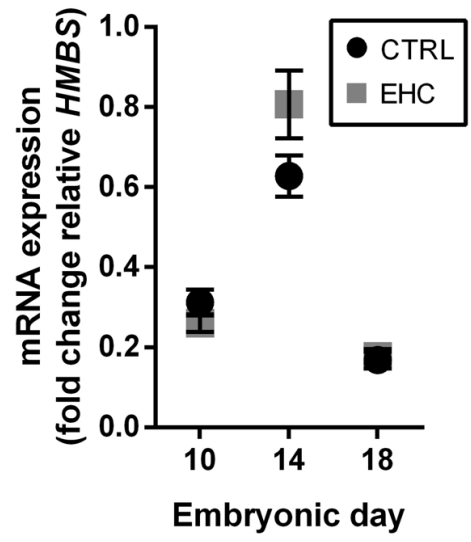

G DNMT3B

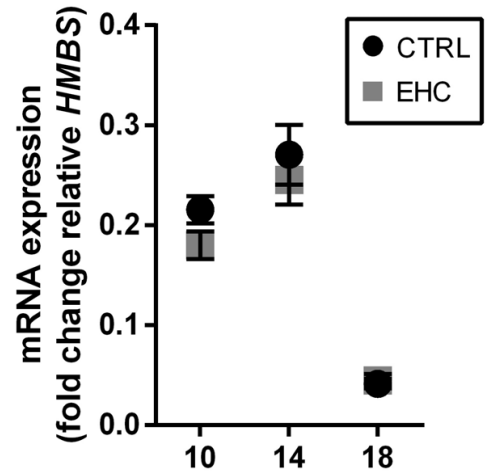

Embryonic day

FIGURE 2 | TET family enzymes present increased expression during EHC. (A) Experimental scheme. Midbrain samples were taken on embryonic days (ED) 10 and 14 (during conditioning), and on ED 18 (after conditioning). (B) mRNA expression of TET1 did not differ between control (CTRL) and EHC groups on ED 10 $\left(\mathrm{n}_{C T R L}=10, \mathrm{n}_{E H C}=10, P=0.18\right), 14\left(\mathrm{n}_{C T R L}=9, \mathrm{n}_{E H C}=8, P=0.08\right)$ or $18\left(\mathrm{n}_{C T R L}=10, \mathrm{n}_{E H C}=10, P=0.7\right)$. (C) mRNA expression of TET2 was significantly lower in the EHC vs. CTRL group on ED $10\left(\mathrm{n}_{C T R L}=10, \mathrm{n}_{E H C}=10,{ }^{\star \star} P=0.006\right)$, but significantly higher on ED $14\left(\mathrm{n}_{C T R L}=9, \mathrm{n}_{E H C}=8,{ }^{*} P=0.03\right)$, and did not differ on ED $18\left(\mathrm{n}_{C T R L}=10, \mathrm{n}_{E H C}=10, P=0.73\right.$ ). (D) mRNA expression of TET3 did not differ between CTRL and EHC groups on ED $10\left(\mathrm{n}_{C T R L}=10, \mathrm{n}_{E H C}=10, P=0.2\right), 14$ $\left(\mathrm{n}_{C T R L}=9, \mathrm{n}_{E H C}=8, P=0.09\right)$ or $18\left(\mathrm{n}_{C T R L}=10, \mathrm{n}_{E H C}=10, P=0.74\right)$. (E) mRNA expression of DNMT1 did not differ between CTRL and EHC groups on ED 10 $\left(\mathrm{n}_{C T R L}=10, \mathrm{n}_{E H C}=10, P=0.07\right), 14$ ( $\mathrm{n}_{C T R L}=9, \mathrm{n}_{E H C}=8, P=0.57$ ) or $18\left(\mathrm{n}_{C T R L}=10, \mathrm{n}_{E H C}=10, P=0.32\right) .(\mathbf{F})$ mRNA expression of DNMT3A was significantly lower in the EHC vs. CTRL group on ED $10\left(\mathrm{n}_{C T R L}=10, \mathrm{n}_{E H C}=10,{ }^{\star} P=0.02\right)$, but did not differ on ED $14\left(\mathrm{n}_{C T R L}=9, \mathrm{n}_{E H C}=8, P=0.09\right)$, and did not differ on ED $18\left(\mathrm{n}_{C T R L}=10, \mathrm{n}_{E H C}=10, P=0.81\right)$. (G) mRNA expression of DNMT3B did not differ between CTRL and EHC groups on ED 10 ( $\left.\mathrm{n}_{C T R L}=10, \mathrm{n}_{E H C}=10, P=0.08\right)$, $14\left(\mathrm{n}_{C T R L}=9, \mathrm{n}_{E H C}=8, P=0.53\right.$ ) or $18\left(\mathrm{n}_{C T R L}=10, \mathrm{n}_{E H C}=10, P=0.71\right)$. Data are presented as mean $\pm \mathrm{SEM}$, and significant effects between groups are indicated by ${ }^{\star} 0.05<P<0.01,{ }^{\star \star} 0.01<P<0.001$. $\mathrm{RH}$, relative humidity. 


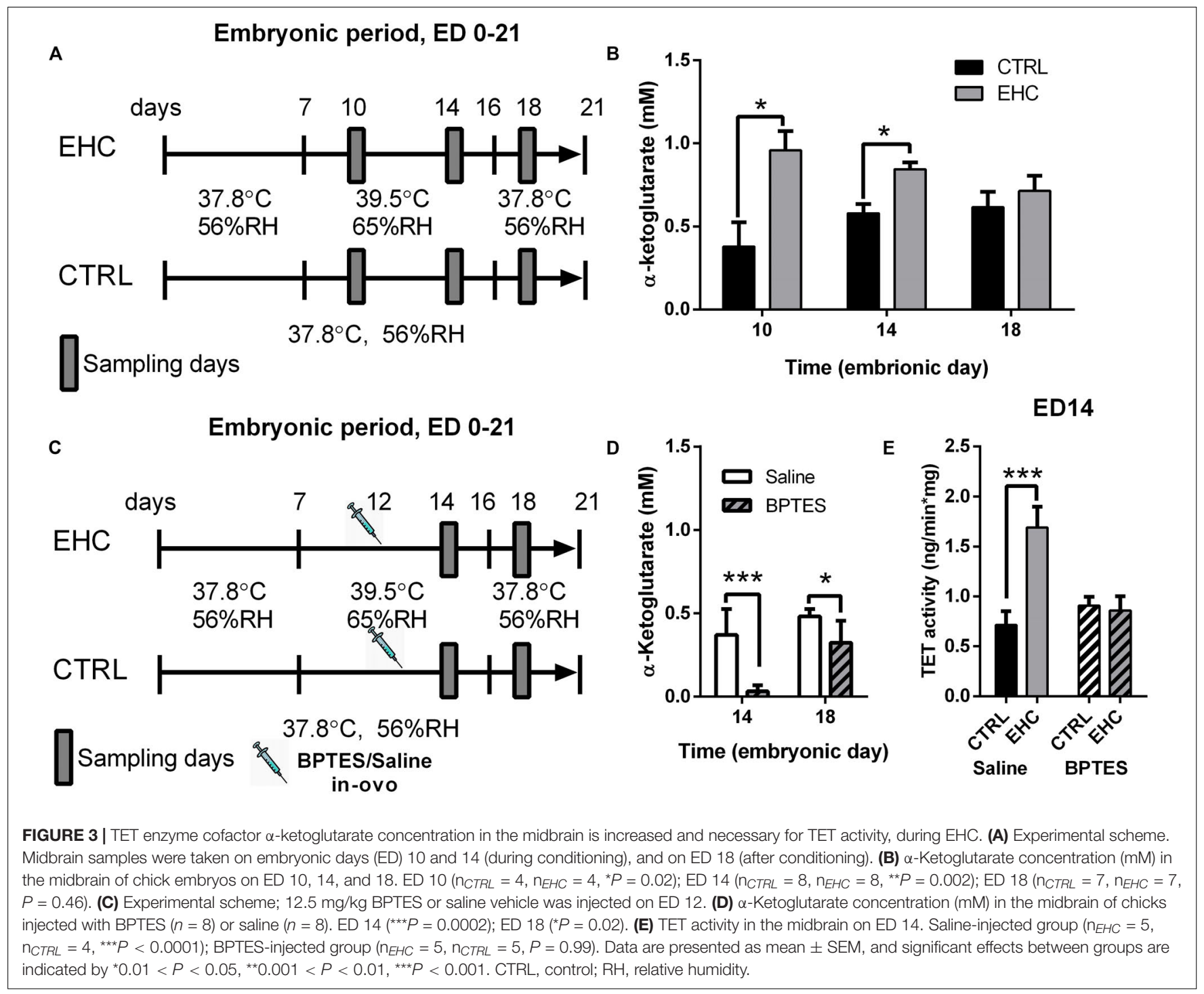

in the embryonic period. In addition to body temperature, we measured mRNA expression, in the hypothalamus $6 \mathrm{~h}$ into the heat challenge, of heat and stress related genes including: HSP70 which is an indicator of the chick's heat sensitivity (Kisliouk et al., 2017); brain derived neurotrophic factor (BDNF) which is epigenetically regulated during heat conditioning (Katz and Meiri, 2006; Kisliouk and Meiri, 2009); glucocorticoidinduced leucine zipper (GILZ), involved in glucocorticoids antiinflammatory effect (Riccardi, 2015); eukaryotic initiation factor $2 \mathrm{~b} 5$ (EIF2B5) involved in the epigenetic regulation of thermal control establishment (Kisliouk et al., 2009); and corticotrophin release hormone receptors $(C R H R) 1$ and 2 which are increased in the hypothalamus of chicks during food deprivation (Kadhim et al., 2019). The expression of each group was normalized to the expression in a parallel $t_{0}$ group which was not subjected to heat challenge. Multiple comparisons showed that increased expression of HSP70 following heat challenge in the saline-injected EHC group was significantly lower $(P=0.048)$ than that in the saline-injected control group. However, HSP70 expression in the BPTES-injected EHC group did not differ from that in the saline-injected controls $(P=0.94)$, and was significantly higher than that in the saline-injected EHC group $(P=0.042)$. There was a trend toward increased expression in the BPTES-injected EHC group compared to the BPTESinjected control group $(P=0.099)$, and a trend toward increased expression in the BPTES-injected control group compared to the saline-injected control group $(P=0.087)$. Although there was a strong BPTES effect $\left(F_{1}, 20=7.902, P=0.01\right)$, it did not interact $\left(F_{1}, 20=0.072, P=0.79\right)$ with the conditioning effect $\left(F_{1}, 20=7.355, P=0.01\right.$; Figure 4G). BDNF expression during heat stress was significantly lower in the saline-injected EHC group compared to the saline-injected control group $(P=0.039)$, however embryonic injection of BPTES attenuated this effect and there was no significant difference in $B D N F$ expression between CTRL and EHC groups injected with BPTES $(P=0.8)$. Although there was a strong conditioning effect $\left(F_{1}, 20=4.889, P=0.04\right)$, 


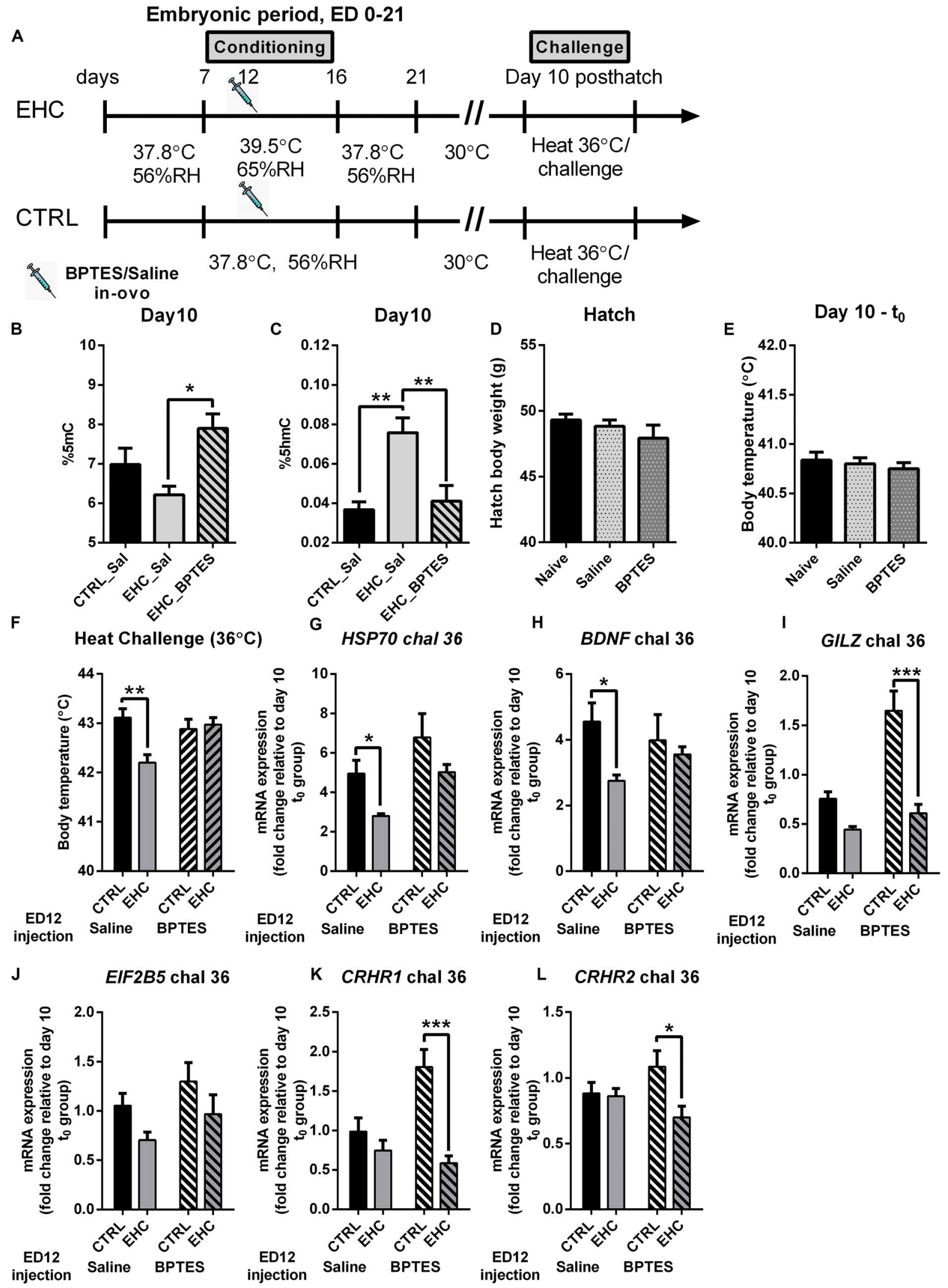




\begin{abstract}
FIGURE 4 | TET inhibition during EHC has a long-lasting effect, blocking heat resilience later in life. (A) Experimental scheme; $12.5 \mathrm{mg} / \mathrm{kg}$ BPTES or saline vehicle was injected on embryonic day (ED) 12, heat challenge was applied on day 10 post-hatch. (B) Total DNA\%5mC in the hypothalamus on day 10 post-hatch, no challenge. EHC groups [ $\left.n_{B P T E S}=6, n_{\text {saline }}(S a l)=5,{ }^{*} P=0.02\right]$; control (CTRL)-Sal group $(n=7$; vs. EHC-Sal group $P=0.24)$. (C) Total DNA\%5hmC in the hypothalamus on day 10 post-hatch, no challenge. EHC groups $\left[n_{B P T E S}=6, n_{\text {saline }}(S a l)=7,{ }^{* *} P=0.006\right]$; CTRL-Sal group $\left(n=7\right.$; vs. EHC-Sal group $\left.{ }^{* \star} P=0.002\right)$. (D) Hatch body weight (g). Naïve (non-injected, $n=75)$, saline-injected group $(n=59)$ and BPTES-injected group $\left(n=51 ; F_{2}, 182=1.216, P=0.299\right)$. (E) Day $10 t_{0}$ (before heat challenge) body temperature. Naïve $(n=8)$, saline-injected group $(n=17)$ and BPTES-injected group $\left(n=12 ; F_{2}, 34=0.365, P=0.697\right)$. (F) Day 10 heat challenge $\left(36^{\circ} \mathrm{C}\right)$. ED 12 saline-injected groups $\left(\mathrm{n}_{C T R L}=8, \mathrm{n}_{E H C}=7,{ }^{* \star} P=0.004\right)$; ED 12 BPTES-injected groups $\left(\mathrm{n}_{C T R L}=6, \mathrm{n}_{E H C}=7, P=0.99\right) ; E H C-B P T E S$ group vs. EHC-saline group ( $\left.{ }^{*} P=0.02\right)$. (G) Hypothalamic mRNA expression of HSP70 during day 10 heat challenge, normalized to HMBS and reported relative to their respective $t_{0}$ controls. Groups injected with saline on $E D 12\left(n_{E H C}=6, n_{C T R L}=6,{ }^{*} P=0.048\right)$; groups injected with BPTES on ED 12 ( $n_{E H C}=6, n_{C T R L}=6$, $P=0.099)$. (H) Hypothalamic mRNA expression of BDNF during day 10 heat challenge, normalized to HMBS and reported relative to their respective $t_{0}$ controls. Groups injected with saline on ED 12 ( $\left.n_{E H C}=6, n_{C T R L}=6,{ }^{*} P=0.039\right)$; groups injected with BPTES on ED $12\left(n_{E H C}=6, n_{C T R L}=6, P=0.80\right)$. (l) HypothalamiC mRNA expression of GILZ during day 10 heat challenge, normalized to HMBS and reported relative to their respective to controls. Groups injected with saline on ED $12\left(\mathrm{n}_{E H C}=6, \mathrm{n}_{C T R L}=6, P=0.137\right)$; groups injected with BPTES on ED $12\left(\mathrm{n}_{E H C}=6, \mathrm{n}_{C T R L}=6,{ }^{* * *} P<0.001\right)$. (J) Hypothalamic mRNA expression of EIF2B5 during day 10 heat challenge, normalized to $H M B S$ and reported relative to their respective $t_{0}$ controls. Groups injected with saline on $E D 12\left(n_{E H C}=6, n_{C T R L}=6\right.$, $P=0.245)$; groups injected with BPTES on ED $12\left(n_{E H C}=6, n_{C T R L}=6, P=0.277\right)$. (K) Hypothalamic mRNA expression of CRHR1 during day 10 heat challenge, normalized to HMBS and reported relative to their respective to controls. Groups injected with saline on $E D 12\left(\mathrm{n}_{E H C}=6, \mathrm{n}_{C T R L}=6, P=0.523\right)$; groups injected with BPTES on ED $12\left(n_{E H C}=6, n_{C T R L}=6,{ }^{* \star *} P<0.001\right)$. (L) Hypothalamic mRNA expression of CRHR2 during day 10 heat challenge, normalized to HMBS and reported relative to their respective $t_{0}$ controls. Groups injected with saline on ED 12 ( $n_{E H C}=6, n_{C T R L}=6, P=0.980$ ); groups injected with $B P T E S$ on ED 12 $\left(\mathrm{n}_{E H C}=6, \mathrm{n}_{C T R L}=6,{ }^{*} P=0.012\right)$. Data are presented as mean $\pm \mathrm{SEM}$. Significant effects between groups are indicated by ${ }^{*} 0.05<P<0.01,{ }^{*} 0.01<P<0.001$ using ANOVA test with Sidak's multiple comparisons test. $\mathrm{RH}$, relative humidity.
\end{abstract}

there was no BPTES effect $\left(F_{1}, 20=0.05, P=0.82\right)$ nor interaction $\left(F_{1}, 20=1.86, P=0.19\right.$; Figure $\left.4 \mathrm{H}\right)$. GILZ expression during heat stress was significantly lower in the BPTES-injected EHC group compared with the BPTES-injected CTRL group $(P<0.001)$, this reduction was not significant between the saline-injected control and EHC groups $(P=0.14)$. Nevertheless, there was a significant interaction $\left(F_{1}, 20=9.68, P=0.005\right)$, along with BPTES effect $\left(F_{1}, 20=20.65, P<0.001\right)$ as well as conditioning effect $\left(F_{1}, 20=33.71, P<0.001\right.$; Figure 4I). EIF2B5 expression during heat stress did not differ between saline-injected CTRL and EHC groups $(P=0.24)$, nor between BPTES-injected CTRL and EHC groups $(P=0.28)$. Nevertheless, both saline and BPTES-injected EHC groups presented a tendency toward reduced expression compared to their controls, presenting a significant conditioning effect $\left(F_{1}, 20=4.71, P=0.04\right.$; Figure 4J). CRHR1 expression during heat stress was significantly lower in the BPTES-injected EHC group compared with BPTES-injected CTRL group $(P<0.001)$, but there was no difference between saline-injected CTRL and EHC groups $(P=0.52)$. Overall, there was a strong interaction $\left(F_{1}, 20=9.21, P=0.007\right)$ along with significant conditioning effect $\left(F_{1}, 20=20.34, P<0.001\right)$ and a trend for BPTES effect $\left(F_{1}, 20=4.15, \mathrm{P}=0.055\right.$; Figure $\left.4 \mathrm{~K}\right)$. CRHR2 expression during heat stress was significantly lower in the BPTES-injected EHC group compared with BPTES-injected CTRL group $(P=0.012)$, but there was no difference between saline-injected CTRL and EHC groups $(P=0.98)$. There was a significant conditioning effect $\left(F_{1}, 20=5.24, P=0.033\right)$ along with a trend for interaction $\left(F_{1}, 20=4.13, P=0.056\right.$; Figure 4L).

\section{TET Inhibition During EHC Also Blocks Attenuation of the Inflammatory Response in the Hypothalamus, Later in Life}

Both the BPTES- and saline-injected groups were challenged with LPS on day 10 post-hatch (Figure 5A). The groups injected with BPTES (Figure 5B, right panel) presented a significant increase in body temperature in both the control $(P<0.0001)$ and EHC $(P=0.0006)$ groups $6 \mathrm{~h}$ into LPS challenge, but there was no difference in body temperature between the controland EHC-BPTES groups during LPS challenge $(P=0.99)$. In addition, BPTES-injected groups presented only the LPS effect $\left(F_{1}, 22=72.54, P<0.0001\right)$, but no conditioning effect $\left(F_{1}\right.$, $22=0.77, P=0.39)$ or interaction $\left(F_{1}, 22=2.24, P=0.15\right)$. The groups that were injected with saline on ED 12 (Figure 5B, left panel), and challenged with LPS on day 10 post-hatch (19 days later) showed that although there was a significant increase in body temperature in both the EHC $(P=0.026)$ and control $(P=0.003)$ groups, the control group's body temperature was significantly higher than that of the EHC group $(P=0.032)$, presenting a significant LPS effect $\left(F_{1}, 23=26, P<0.001\right)$, as well as a conditioning effect $\left(F_{1}, 23=12.5, P=0.002\right)$, with no interaction $\left(F_{1}, 23=0.53, P=0.47\right)$. These results indicated that while saline injection on ED 12 does not impair heat or inflammatory resilience, BPTES impairs both.

Next, we analyzed the influence of BPTES on proinflammatory (LITAF, NFאB, NOS2, and VEGFA) and anti-inflammatory (MRC1 and IL10) gene expression, $6 \mathrm{~h}$ into LPS challenge (Figures 5C-F). BPTES had no effect on IL10 or MRC1 expression (data not shown). The reduction in LITAF expression in the saline-injected EHC group compared to the saline-injected controls $(P=0.03)$ was not evident in the BPTES-injected groups $(P=0.68$; Figure $5 \mathrm{C})$. Similarly, the reduction in $N F \kappa B$ expression in the EHC group compared to the saline-injected controls $(P=0.005)$ did not appear in the BPTES-injected groups $(P=0.25)$. Moreover, the BPTES-injected EHC group presented higher expression of $N F \kappa B$ compared to the salineinjected EHC group $(P<0.0001$; Figure 5D). NOS2 expression, following LPS challenge, was reduced in the saline-injected EHC group compared to the saline-injected unconditioned controls $(P=0.02)$. However, there was no difference between the BPTESinjected control and EHC groups $(P=0.89)$, which displayed high expression similar to unconditioned saline injected controls. Two-way ANOVA revealed a significant interaction between the conditioning and BPTES effects $\left(F_{1}, 20=5.067, P=0.04\right.$; 
A

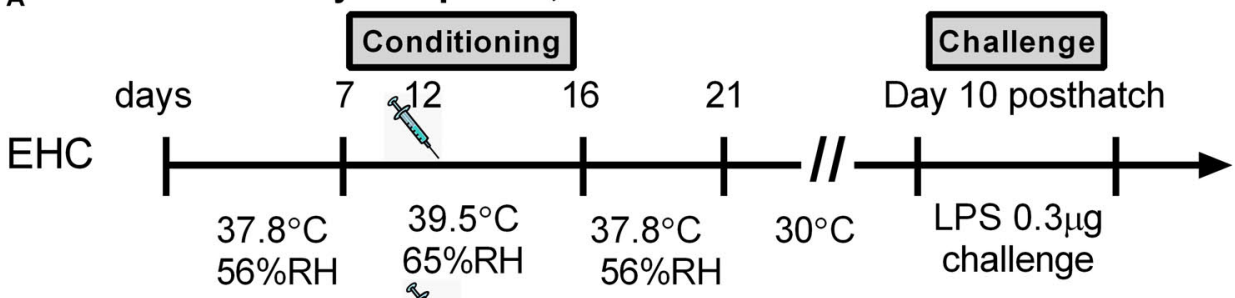

CTRL

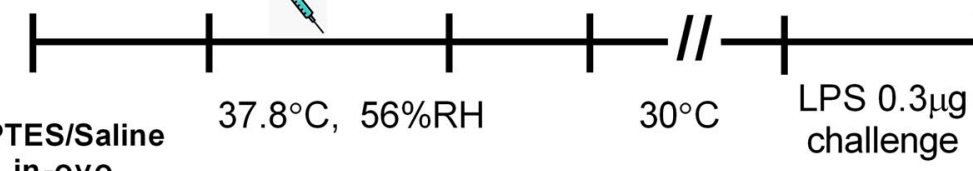

LPS Challenge

B

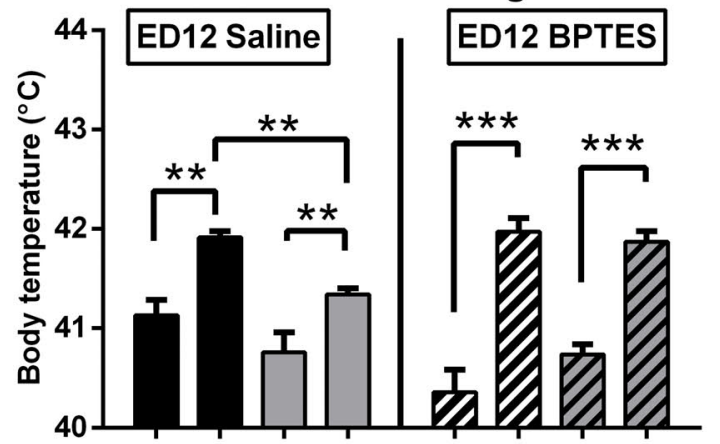

Day 10 injection

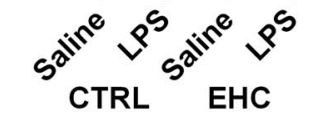

challenge

\section{LITAF day 10}

C

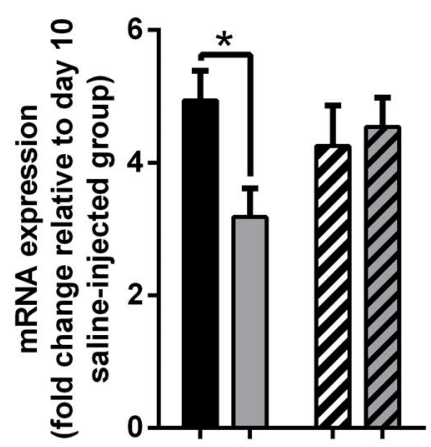

ED12 injection

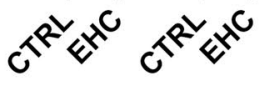

D

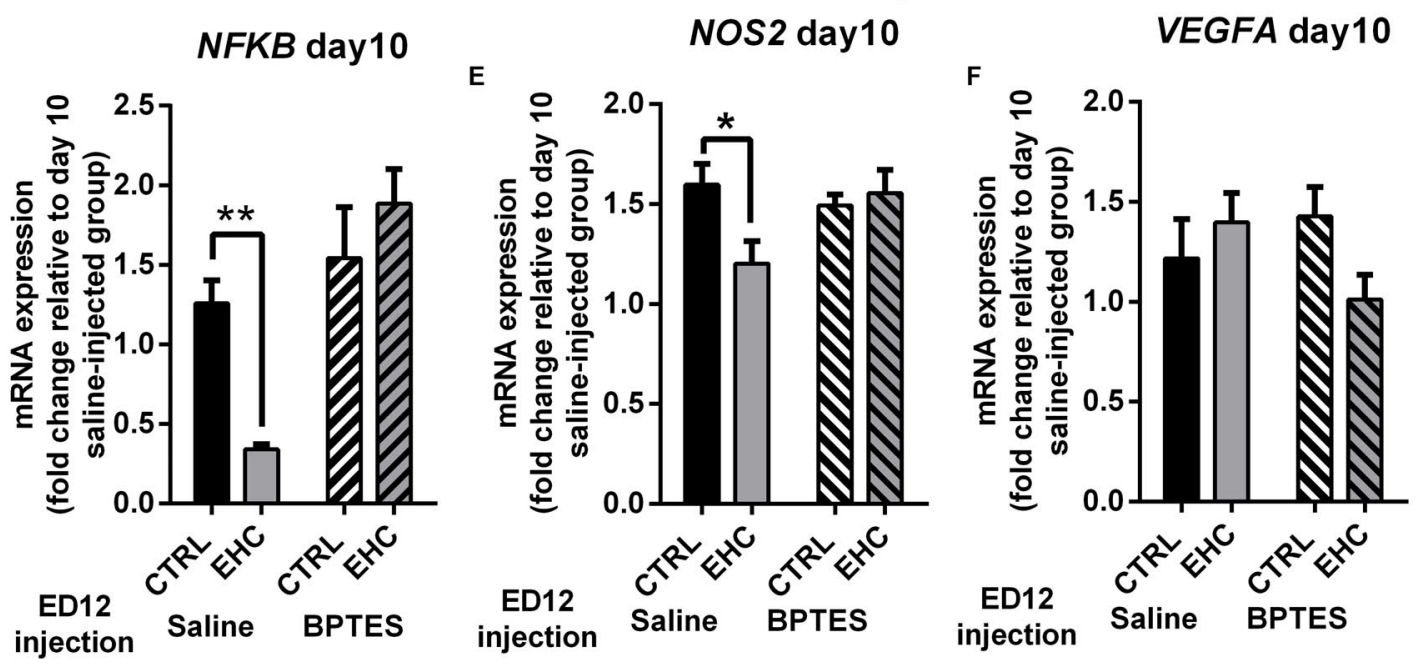

FIGURE 5 | TET inhibition during EHC also blocks attenuation of inflammatory response in the hypothalamus, later in life. (A) Experimental scheme; $12.5 \mathrm{mg} / \mathrm{kg}$ BPTES or saline vehicle was injected on embryonic day (ED) 12, LPS challenge was applied on day 10 post-hatch. (B) LPS challenge on day 10 post-hatch. Groups injected with saline on ED 12 (left panel). Control (CTRL) groups ( $\mathrm{n}_{L P S}=6, \mathrm{n}_{\text {saline }}=7,{ }^{\star \star} P=0.003$ ); EHC groups $\left(\mathrm{n}_{L P S}=7, \mathrm{n}_{\text {saline }}=7,{ }^{\star \star} P=0.006\right) ; \mathrm{LPS}-\mathrm{EHC}$ group vs. LPS-CTRL group ( $\left.{ }^{* *} P=0.007\right)$. Groups injected with BPTES on ED 12 (right panel). CTRL groups $\left(n_{L P S}=7, n_{\text {saline }}=7,{ }^{* \star *} P<0.0001\right) ; E H C$ groups $\left(n_{L P S}=7\right.$, $\left.\mathrm{n}_{\text {saline }}=5,{ }^{\star \star \star} P<0.0001\right)$; EHC-LPS group vs. EHC-saline group $(P=0.62)$. (C-F) Hypothalamic mRNA expression was measured on day 10 post-hatch, $6 \mathrm{~h}$ after LPS or saline injection; fold change of LITAF, NFKB, NOS2, and VEGFA was normalized to HMBS and reported relative to their saline controls. (C) LITAF. Groups injected with saline on $E D 12\left(\mathrm{n}_{E H C}=5, \mathrm{n}_{C T R L}=6,{ }^{*} P=0.03\right)$; groups injected with BPTES on ED $12\left(\mathrm{n}_{E H C}=6, \mathrm{n}_{C T R L}=6, P=0.68\right)$. (D) NFKB. Groups injected with saline on ED $12\left(n_{E H C}=6, n_{C T R L}=6,{ }^{*} P=0.005\right)$; groups injected with BPTES on ED 12 ( $\left.n_{E H C}=6, n_{C T R L}=6, P=0.25\right)$. (E) NOS2. Groups injected with saline on ED $12\left(n_{E H C}=6, n_{C T R L}=6,{ }^{*} P=0.024\right)$; groups injected with BPTES on ED $12\left(n_{E H C}=6, n_{C T R L}=6, P=0.895\right)$. (F) VEGFA. Groups injected with saline on ED 12 $\left(\mathrm{n}_{E H C}=6, \mathrm{n}_{C T R L}=6, P=0.667\right)$; groups injected with BPTES on ED $12\left(\mathrm{n}_{E H C}=6, \mathrm{n}_{C T R L}=6, P=0.144\right)$. Data are presented as mean $\pm S E M$. Significant effects between groups are indicated by ${ }^{\star} 0.05<P<0.01,{ }^{\star \star} 0.01<P<0.001,{ }^{\star \star \star} P<0.0001$ using ANOVA Sidak's multiple comparisons test. RH, relative humidity. 
Figure 5E). VEGFA expression, did not differ between groups, and was not affected by conditioning or BPTES injection (Figure 5F). Overall, BPTES inhibition of TET activity interfered with the EHC-induced cross-tolerance to inflammation via upregulation of the expression of the proinflammatory genes $L I T A F, N F \kappa B$, and NOS2.

\section{Locus Specific Hydroxymethylation and Methylation, in 10 Days-Old Chicks Is Dependent on Embryonic TET Activity}

We found that embryonic inhibition of TET reduces total DNA hydroxymethylation, and influences the expression of proinflammatory genes as well as stress related genes in the hypothalamus. Therefore, we wanted to see if the changes in gene expression is supported by changes in locus specific DNA hydroxymethylation $(\% 5 \mathrm{hmC})$ and methylation $(\% 5 \mathrm{mC})$. To this end, we extracted DNA from the anterior hypothalamus of 10 day-old unchallenged cheeks that were either embryonically conditioned (EHC) or unconditioned and were injected with either BPTES or saline on ED 12 (Figure 6A), to study locus specific hydroxymethylation and methylation. Since we found TET dependent LITAF, NFkB, and GILZ changes in expression, during LPS or heat challenge, we chose these targets to assess locus specific hydroxymethylation and methylation. Genetic areas presenting possible transcriptional activity by the TFBIND algorithm, as well as enriched in CCGG motif, were chosen for analysis. LITAF CpG hydroxymethylation $(\% 5 \mathrm{hmC})$ and methylation $(\% 5 \mathrm{mC})$ was measured between NC_006101.5 461-645 (relative to transcription start), as this region contains 5 CCGG motifs as well as $126 \mathrm{TF}$ binding sites with over $80 \%$ binding probability (Figures 6B,C). LITAF locus specific hydroxymethylation $(\% 5 \mathrm{hmC})$, was significantly increase in the saline-injected EHC group compared with saline injected controls $(P<0.0001)$, embryonic injection of BPTES, blocked hydroxymethylation, and both EHC and control groups presented reduced hydroxymethylation with no significant difference between groups $(P=0.52)$. Twoway ANOVA revealed a strong interaction $\left(F_{1}, 20=14.48\right.$, $P=0.001)$, along with conditioning $\left(F_{1}, 20=27.98, P<0.0001\right)$ and $\operatorname{BPTES}\left(F_{1}, 20=14.22, P=0.001\right)$ effects (Figure 6B). LITAF Locus specific methylation $(\% 5 \mathrm{mC})$ was not affected by BPTES, with significant conditioning effect $\left(F_{1}, 20=7.96\right.$, $P=0.01$; Figure 6C). NFkB CpG hydroxymethylation (\%5hmC) and methylation $(\% 5 \mathrm{mC})$ was measured between NC_006093.5 78-200 (relative to transcription start), as this region contains 2 CCGG motifs as well as 75 TF binding sites with over $80 \%$ binding probability (Figures 6D,E). NFkB locus specific hydroxymethylation $(\% 5 \mathrm{hmC})$, was significantly increased in the saline-injected EHC group compared with saline-injected controls $(P=0.037)$. Embryonic injection of BPTES had a long lasting effect reducing\% $5 \mathrm{hmC}$ in both BPTES-injected groups, with no difference between control and EHC groups $(P=0.9)$. Moreover, BPTES-injected control group displayed $0 \% 5 \mathrm{hmC}$, in all subjects. Two-way ANOVA presented a conditioning $\left(F_{1}, 20=4.43, P=0.048\right)$ as well as a strong BPTES effect $\left(F_{1}, 20=14.61, P=0.001\right.$; Figure 6D). NFkB locus specific methylation $(\% 5 \mathrm{mC})$, was not affected by conditioning nor BPTES (Figure 6E). GILZ locus specific hydroxymethylation $(\% 5 \mathrm{hmC})$ and methylation $(\% 5 \mathrm{mC})$ was measured between NC_006091.5 4045-4248 (relative to transcription start), as this region contains 4 CCGG motifs as well as $126 \mathrm{TF}$ binding sites with over $80 \%$ binding probability (Figures 6F,G). GILZ\%5hmC did not differ between control and EHC salineinjected groups, there was also no difference between control and EHC BPTES-injected groups. However, BPTES injected groups presented reduced $\% 5 \mathrm{hmC}$, than saline injected groups and the effect of BPTES was significant $\left(F_{1}, 20=11.13, P=0.003\right.$; Figure 6F). GILZ locus specific methylation $(\% 5 \mathrm{mC})$, was not affected by conditioning nor BPTES (Figure 6G). Overall, the general effect of BPTES embryonic injection reducing total hydroxymethylation is also evident in specific targets along the TLR4/MyD88 inflammatory signaling pathway.

\section{DISCUSSION}

Early life stress can affect an organism's future response to stress (Taylor, 2010). For example, according to Barker's hypothesis, poor embryonic development leads to lifelong adversities (Barker, 1990; Hales and Barker, 1992). In this work, we focused on manipulations of the embryonic environment leading to stress cross-tolerance later in life. Cross-tolerance is a beneficial side effect of resilience of a completely different nature to another conditioned stressor (Horowitz, 2017). Here we demonstrated that EHC has long-term effects on inflammatory resilience in the hypothalamus. Furthermore, we revealed that heat-inflammatory cross-tolerance depends on the epigenetic increase of TET transcription and activity that occurs during the embryonic thermal manipulation and persists throughout life.

Across different species, heat acclimation has been shown to protect against a wide range of stressors (Shein et al., 2008; Umschwief et al., 2010, Umschweif et al., 2014; Assayag et al., 2012; Horowitz, 2017; Pollak et al., 2017; Hossain et al., 2018). Various findings point to the hypothalamus as the site of convergence of thermal-inflammation-related networks (Tzschentke, 2007; Tona et al., 2008; Tzschentke and Halle, 2009; Hueston and Deak, 2014; Loyau et al., 2014, 2016; Chao et al., 2015; Klett et al., 2015; Nassar et al., 2015; Audet et al., 2016; Morrison, 2016).

To demonstrate the concept of heat-conditioning leading to cross-tolerance involving the inflammatory response, we performed an LPS challenge on day 10 post-hatch by direct ICV injection of LPS into the brain. This approach was used so that the LPS effect would be directed to the hypothalamus, rather than to peripheral inflammation. The TLR4/MyD88 pathway was chosen to prove inflammatory resilience in the hypothalamus, because it has been previously shown to be induced during hypothalamic inflammation (Ropelle et al., 2010; Macrez et al., 2011; Valdearcos et al., 2015).

Therefore, we analyzed the mRNA expression of LITAF and $N F \kappa B$ as proinflammatory markers, following LPS injection. We found, $6 \mathrm{~h}$ into the LPS challenge, induced febrile response and overexpression of $L I T A F$ and $N F K B$, in addition to NFKB 
A

Embryonic period, ED 0-21
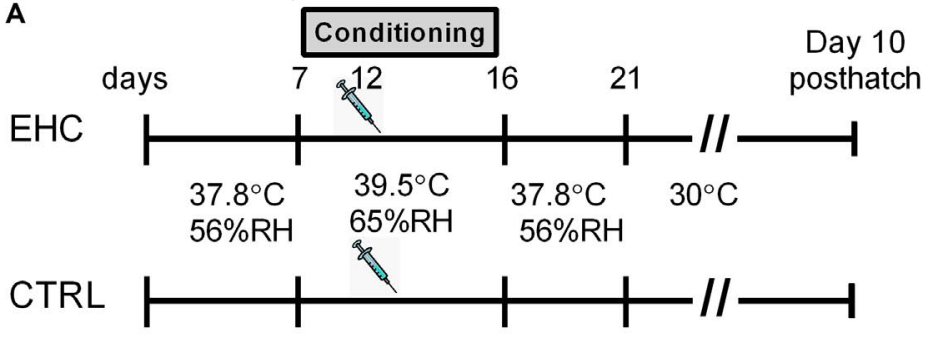
BPTES/Saline
in-ovo
$37.8^{\circ} \mathrm{C}, 56 \% \mathrm{RH} \quad 30^{\circ} \mathrm{C}$

LITAF day 10

B
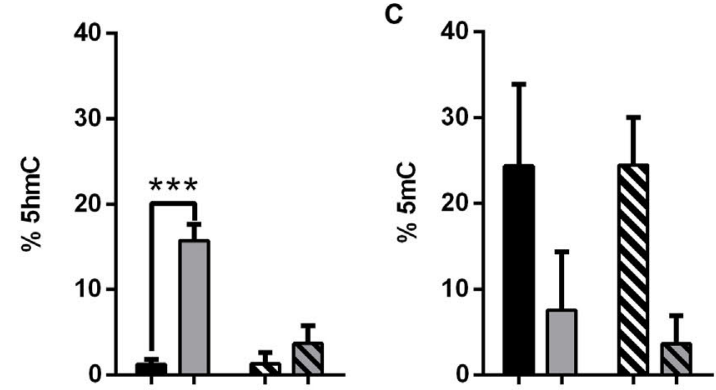

ED12
injection
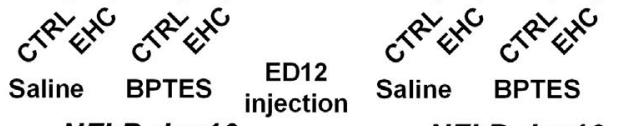

NFkB day10

NFkB day10

D
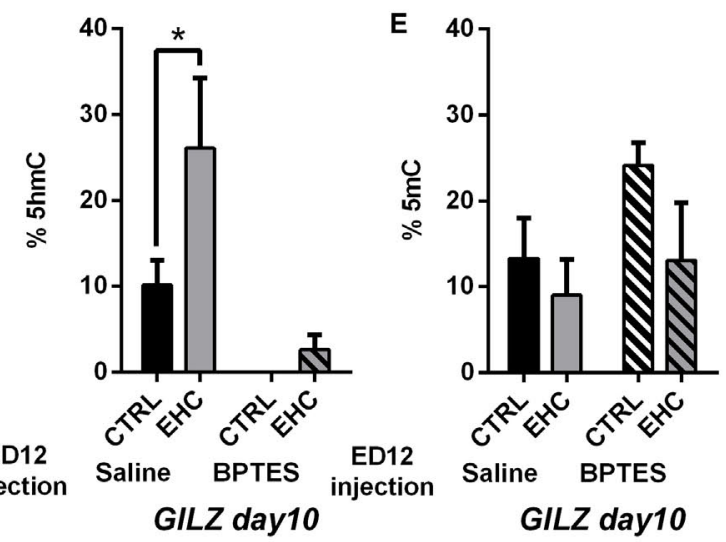

$\mathbf{F}$

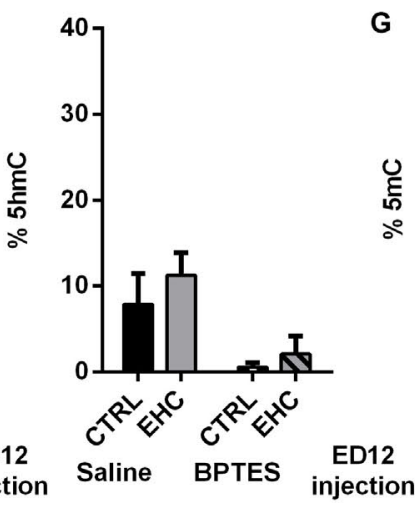

G

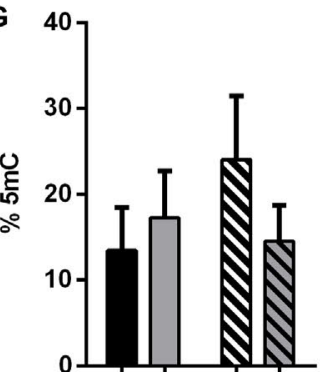

injection

FIGURE 6 | Continued 


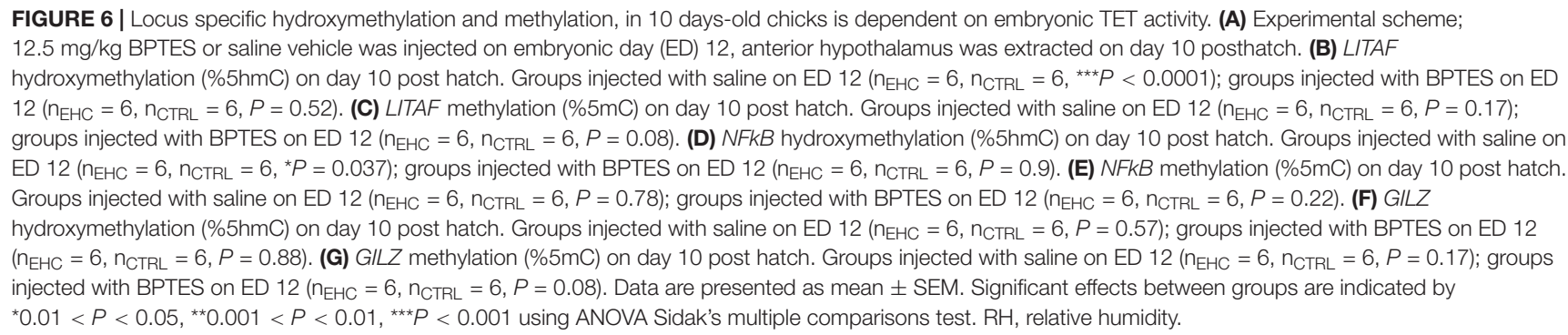

activation and nuclear translocation in the hypothalamus, all of which occur naturally during ischemic and high-fatdiet-induced hypothalamic inflammation (Macrez et al., 2011; Valdearcos et al., 2015). However, all of these effects were significantly reduced in the EHC group, along with increased expression of the anti-inflammatory genes $I L 10$ and $M R C 1$. In the CNS, LPS binds to the TLR4 receptor and this activation is associated with neurodegenerative diseases (Xiang et al., 2015) and major depressive disorder (MDD). Furthermore, acute TLR4 stimulation activates the HPA axis (Liu et al., 2014), as well as being activated by alcohol binging (Crews et al., 2015). In chicks, HPA axis activation can be induced by heat-stress (Cramer et al., 2015), and indeed we see that LPS administration induces an increase in the chick's body temperature, but to a lesser extent in the EHC model.

In this work, we studied the expression of both TETs and DNMTs during and after conditioning. Since we found TET2 expression to be significantly increased in the EHC group, compared with controls, during conditioning (on ED 14), we chose to focus here on the role of TET family enzymes during EHC on the establishment of inflammatory resilience later in life. In a previous study, regarding the involvement of TET2 in cardiovascular disease, TET2 knockouts presented increased macrophage inflammatory activation, accelerating atherosclerosis (Fuster et al., 2017). Moreover, both TET and DNMT expression presented elevated levels during conditioning, which plummeted back to baseline after the conditioning period, suggesting that embryonic heat conditioning occurs at a time susceptible toward epigenetic modifications. Interestingly, DNMT3A, previously found to regulates TET2 and TET3 expression (Gong et al., 2016), presented increased expression both in the EHC and unconditioned groups, however, TET2 and TET3 expression was still elevated in both groups, during conditioning. This observation may suggest that although DNMT's expression is elevated during EHC, TETs expression is more prominent. Both TET family enzymes and DNMT's are regulated by metabolic intermediates such as $\alpha$-keto-glutarate, S-adenosylmethionine (SAM), and Acetyl-CoA (Baardman et al., 2015), therefore epigenetic modifications are sensitive to metabolic regulation. Furthermore, embryonic heat conditioning exhibits long lasting metabolic effects in the fully grown chicken (Piestun et al., 2008, 2009).

In addition to our observations, previous studies have demonstrated that early life thermal conditioning induces stress resilience via the activation of TET enzymes (Cramer et al., 2018, 2019). Indeed, we found that EHC causes increased transcription of TET1 and TET2, during conditioning, compared to controls. TET3 expression was increased during the conditioning period in both groups, indicating its possible role during this developmental period. Moreover, TET2 expression was significantly lower in the EHC group compared to controls at the beginning of the conditioning period (ED 10), but its enhanced transcription led to significantly higher expression on ED 14. In a recent study, TET expression in the hypothalamus was shown to be reduced in mice after birth (Cisternas et al., 2020), indicating a possible embryonic role.

These dynamic changes in TET family enzyme expression during the conditioning period led us to further study their involvement in the creation of EHC-induced cross-tolerance to inflammation. Interestingly, TET family enzymes have been previously reported to regulate different inflammatory events: e.g., activating inflammatory genes during macrophage differentiation and monocyte activation in monogenic inflammatory syndromes (Vento-Tormo et al., 2017); resolving inflammation in human peripheral blood mononuclear cells and murine peritoneal macrophages (Zhang et al., 2015); inducing cytokine expression in human dental pulp cells (Wang et al., 2018); and exhibiting TET-dependent anti-inflammatory cytokine regulation in human PMA-differentiated THP-1 cells and human primary mononuclear cells (Hassan et al., 2018), and adaptive immunity in zebrafish (Yang et al., 2016). However, little is known about the TET family enzymes' role in regulating CNS inflammation. Nevertheless, the TET family enzyme cofactor $\alpha$-ketoglutarate has been shown to be necessary for macrophage anti-inflammatory activation (Liu et al., 2017), for extracellular vesicle release during neuroinflammation (Wu et al., 2018) and also histone lysine demethylation (Niu et al., 2015). Therefore, we decided to measure the concentration of $\alpha$-ketoglutarate in the midbrains of chick embryos during conditioning, and found it to be higher in the EHC group than controls. We then used BPTES to inhibit $\alpha$-ketoglutarate synthesis. Reduced concentration of $\alpha$-ketoglutarate in the midbrain was evident 2 and 6 days after BPTES injection, indicating TET inhibition from the moment of injection (ED 12) through the rest of the conditioning period. To assert BPTES inhibition of not only $\alpha$-ketoglutarate production, but also TET activity, we measured TET activity in the midbrain during conditioning (ED 14). Indeed, we found that TET activity was higher in the salineinjected EHC group than the saline-injected controls, but also that the BPTES-injected EHC group did not present this increase 
in activity, which was similar to that in the BPTES-injected controls. After establishing TET inhibition by BPTES during the embryonic period, we studied the long-term effect of TET inhibition on day 10 post-hatch.

Since TET family enzymes are responsible for active demethylation of methylated CpGs (5mC) (Shi et al., 2017), we measured the total $\% 5 \mathrm{mC}$ on day 10 post-hatch (almost 3 weeks after BPTES injection). The long-term effect of BPTES was evidenced by the higher abundance of the TET substrate $\% 5 \mathrm{mC}$ in BPTES-injected chicks. Moreover, the TET product $(\% 5 \mathrm{hmC})$ in the BPTES-injected EHC group was lower on day 10 posthatch than in the saline-injected EHC chicks, and did not differ from that in the saline-injected controls. This indicated that TET activity during the embryonic period has a long-term effect, which is still evident later in life.

Glutaminolysis is a general process implicating various cellular signaling pathways, among them glycolysis, and its inhibition influences embryonic metabolism (Vernardis et al., 2017). Therefore, we measured the chicks' body weight at hatch, and found no significant differences between BPTESinjected, saline-injected and naive (non-injected) chicks. Furthermore, during heat acclimation, glutamate receptors in the hypothalamus are necessary for the development of ischemic cross-tolerance (Yacobi et al., 2014; Matsuzaki et al., 2017). To ascertain that our results were a direct outcome of TET inhibition, and not of impaired hypothalamic development, we also measured baseline body temperature, prior to heat challenge $\left(t_{0}\right)$ and found no significant difference between groups. To further study the role of TET during the establishment of thermal resilience, we measured the chicks' body temperature $6 \mathrm{~h}$ into the heat challenge and found that BPTES blocks heat resilience. It is also worth noting that BPTES did not change the body temperature of the challenged controls, indicating that the absence of thermal resilience in the BPTESinjected EHC group was a result of TET inhibition, rather than impaired glutamatergic transmission. Moreover, HSP70, shown to be a heat-stress-related marker (Kisliouk et al., 2017), presented higher expression in the BPTES-injected EHC group than in their saline-injected counterparts. We also found that both embryonic TET inhibition and embryonic conditioning affected the expression of the stress related genes GILZ, CRHR1 and CRHR2, during heat challenge. The expression of $B D N F$ and EIF2B5, which were previously shown to be epigenetically regulated during postnatal thermal establishment (Katz and Meiri, 2006; Yossifoff et al., 2008; Kisliouk and Meiri, 2009; Kisliouk et al., 2009), were affected during heat challenge by the embryonic conditioning but not by TET inhibition.

Finally, considering the effect of embryonic TET inhibition on the cross-tolerance to inflammation, we measured the chicks' body temperature during LPS challenge; we found that EHC chicks injected with BPTES do not develop inflammatory crosstolerance, as they displayed increased body temperature, and did not present the reduced expression of the proinflammatory genes LITAF, NFkB and NOS2 found in saline-injected EHC chicks. VEGFA was not affected by the conditioning nor by TET inhibition.
To further study the specific effect of embryonic TET inhibition, in relation to the changes we found in gene expression later in life, we performed locus specific DNA methylation and hydroxymethylation analysis. To asses long-term effects, the analysis was performed on day 10 post-hatch on potential regulatory areas enriched with CpG's that presented increased probability to bind transcription factors. Indeed, embryonic inhibition of TET is not transient, and has a long term effect reducing hydroxymethylation later in life. It should be noted that, reduction in hydroxymethylation was accompanied by an increase in methylation of the same locus. Nevertheless, this effect was less significant probably, due to the activity of DNMT's.

Therefore, we suggest that TET-dependent epigenetic mechanisms that take place during the embryonic conditioning period downregulate inflammatory expression later in life.

The effect of TET family enzymes on inflammation is quite variable and still under investigation. Whereas there are studies showing specific TET-dependent increases in expression of the inflammatory cytokine IL6, as well as demethylation-dependent inflammation (Vento-Tormo et al., 2017; Itoh et al., 2018), others report TET-dependent reduction of inflammation and inflammatory cytokine expression via demethylation or histone deacetylation (Zhang et al., 2015; Chen et al., 2017; Hassan et al., 2018). Nevertheless, our findings of TET-dependent heat and inflammatory cross-tolerance further support our hypothesis that epigenetic mechanisms in the hypothalamus act, upon EHC, to create tolerance to inflammation. It is also worth noting that the effects on TET products and substrates, as well as locus specific effects and resilience, were measured 19 days after glutaminase inhibition, indicating a longterm effect.

This TET-dependent inflammatory resilience is supported by previous findings of inhibition of DNA methylation impairing the LPS-induced inflammatory response in mice (Matt et al., 2018). Furthermore, heat resilience in chicks induced by thermal conditioning on day 3 post-hatch was attenuated by the use of PARP inhibitor, which also inhibits TET family enzymes and reduces DNA hydroxymethylation (Cramer et al., 2019). Moreover TET activity is associated with oxygen metabolism (Matt et al., 2018; Skiles et al., 2018), and treating rats with hyperbaric oxygen has been found to protect against hypothalamic ischemia and inflammation caused by heat stroke (Tai et al., 2010), perhaps via induction of TET activity.

The main goal of this manuscript was to provide evidence that TET activity during heat conditioning at the embryonic period, is both necessary and sufficient to induce resilience to both thermal and inflammatory phenotypes later in life. The repertoire of proteins which are affected is probably very wide. Here, only for the proof of concept and to strengthen the phenotypic effect, we demonstrated a change in the expression of stress and thermal establishment related genes: HSP70, BDNF, GILZ, EIF2B5, CRHR1, and CRHR2 as well as inflammatory-related genes i.e., LITAF, NFkB, NOS2, and VEGFA. It is intriguing to know the effect of embryonic heat conditioning on the entire genomic landscape, and to further analyze those genes to answer questions such as which target is transiently demethylated and 
which gene target will stay demethylated throughout the life span of the animals. These very interesting questions should be addressed in future research.

To conclude, we show that epigenetic regulation by TET family enzymes is pivotal in the establishment of crosstolerance, by demonstrating that embryonic heat stress induces cross-tolerance to hypothalamic inflammation later in life. Furthermore, TET activity proved to be essential for induction of the protective effect. This phenomenon of early life epigenetic changes should be considered for the prevention of disease later in life.

\section{DATA AVAILABILITY STATEMENT}

The datasets generated for this study are available on request to the corresponding author.

\section{ETHICS STATEMENT}

The animal study was reviewed and approved by the ethics committee of the Volcani Institute.

\section{REFERENCES}

André, C., Guzman-quevedo, O., Rey, C., Rémus-borel, J., Clark, S., Castellanosjankiewicz, A., et al. (2017). Inhibiting microglia expansion prevents dietinduced hypothalamic and peripheral in fl ammation. Diabetes 66, 908-919. doi: $10.2337 / \mathrm{db} 16-0586$

Argaw, A. T., Asp, L., Zhang, J., Navrazhina, K., Pham, T., Mariani, J. N., et al. (2012). Astrocyte-derived VEGF-A drives blood-brain barrier disruption in CNS inflammatory disease. J. Clin. Invest. 122, 2454-2468. doi: 10.1172/ JCI60842

Arvin, B., Nevile, L. F., Barone, F. C., and Feurstein, G. Z. (1996). The role of inflammation and cytokines in brain injury. Neurosci. Biobehav. Rev. 20, 445-452. doi: 10.1016/0149-7634(95)00026-7

Assayag, M., Saada, A., Gerstenblith, G., Canaana, H., Shlomai, R., and Horowitz, M. (2012). Mitochondrial performance in heat acclimation-a lesson from ischemia/reperfusion and calcium overload insults in the heart. Am. J. Physiol. Integr. Comp. Physiol. 303, R870-R881. doi: 10.1152/ajpregu.00155. 2012

Audet, G. N., Dineen, S. M., Quinn, C. M., and Leon, L. R. (2016). Altered hypothalamic inflammatory gene expression correlates with heat stroke severity in a conscious rodent model. Brain Res. 1637, 81-90. doi: 10.1016/j.brainres. 2016.01.048

Baardman, J., Licht, I., de Winther, M. P., and Van den, B. J. (2015). Metabolic epigenetic crosstalk in macrophage activation. Epigenomics 7, 1155-1164.

Barker, D. (1990). The fetal and infant origins of adult disease The womb may be more important than the home. BMJ 301:1111.

Béchade, C., Colasse, S., Diana, M. A., Rouault, M., and Bessis, A. (2014). NOS2 expression is restricted to neurons in the healthy brain but is triggered in microglia upon inflammation. Glia 62, 956-963. doi: 10.1002/glia.22652

Branco, M. R., Ficz, G., and Reik, W. (2012). Uncovering the role of 5hydroxymethylcytosine in the epigenome. Nat. Rev. Genet. 13, 7-13. doi: 10. 1038/nrg3080

Carpenter, L. L., Gawuga, C. E., Tyrka, A. R., Lee, J. K., Anderson, G. M., and Price, L. H. (2010). Association between Plasma IL-6 response to acute stress and early-life adversity in healthy adults. Neuropsychopharmacology 35, 2617-2623. doi: 10.1038/npp.2010.159

Chao, C.-M., Cheng, B.-C., Chen, C.-Y., Lin, M.-T., Chang, C.-P., and Yang, S.-T. (2015). Proteomic analysis of hypothalamic injury in heatstroke rats. Proteomics 15, 1921-1934. doi: 10.1002/pmic.201400492

\section{AUTHOR CONTRIBUTIONS}

TR and NM designed and performed the experiments, generated the figures, interpreted the results, and wrote the manuscript. NM, TK, and SD supervised the project. TK, TC, and DS participated in performing the experiments, provided intellectual expertise, and helped interpret the results. All authors contributed to the article and approved the submitted version.

\section{FUNDING}

This work was supported by the Israel Science Foundation under Grant No. 1646/15. Contribution No. 010/19 from the ARO, Volcani Center, Israel.

\section{ACKNOWLEDGMENTS}

We are grateful to the Volcani Center's chicken farm staff for their dedicated work. We would also like to thank Dr. Yuval Cinnamon for his instruction and guidance regarding mid brain extraction from embryos.

Chen, Q., Yin, D., Zhang, Y., Yu, L., Li, X.-D., Zhou, Z.-J., et al. (2017). MicroRNA29a induces loss of 5-hydroxymethylcytosine and promotes metastasis of hepatocellular carcinoma through a TET-SOCS1-MMP9 signaling axis. Cell Death Dis. 8, e2906. doi: 10.1038/cddis.2017.142

Cisternas, C. D., Cortes, L. R., Bruggeman, E. C., Yao, B., and Forger, N. G. (2020). Developmental changes and sex differences in DNA methylation and demethylation in hypothalamic regions of the mouse brain. Epigenetics 15, 72-84. doi: 10.1080/15592294.2019.1649528

Cramer, T., Kisliouk, T., Yeshurun, S., and Meiri, N. (2015). The balance between stress resilience and vulnerability is regulated by corticotropinreleasing hormone during the critical postnatal period for sensory development. Dev. Neurobiol. 75, 842-853. doi: 10.1002/dneu.22252

Cramer, T., Rosenberg, T., Kisliouk, T., and Meiri, N. (2018). Early-life epigenetic changes along the corticotropin-releasing hormone $(\mathrm{CRH})$ gene influence resilience or vulnerability to heat stress later in life. Mol. Psychiatry 24, 10131026. doi: 10.1038/s41380-018-0280-5

Cramer, T., Rosenberg, T., Kisliouk, T., and Meiri, N. (2019). PARP inhibitor affects long-term heat-stress response via changes in DNA methylation. Neuroscience 399, 65-76. doi: 10.1016/J.NEUROSCIENCE.2018.12.018

Crews, F. T., Sarkar, D. K., Qin, L., Zou, J., Boyadjieva, N., and Vetreno, R. P. (2015). Neuroimmune function and the consequences of alcohol exposure. Alcohol Res. Curr. Rev. 37, 331-351.

Duman, R., Ertekin, T., Duman, R., Vurmaz, A., Köken, T., and Bilir, A. (2018). Comparison of the efficacy of different hydrocortisone-induced cataract models in developing chick embryos. J. Pharm. Res. Int. 21, 1-7. doi: 10.9734/JPRI/ $2018 / 39888$

Emsley, H. C. A., Smith, C. J., Tyrrell, P. J., and Hopkins, S. J. (2008). Inflammation in acute ischemic stroke and its relevance to stroke critical care. Neurocrit. Care 9, 125-138. doi: 10.1007/s12028-007-9035-x

Fagundes, C. P., Glaser, R., and Kiecolt-Glaser, J. K. (2013). Stressful early life experiences and immune dysregulation across the lifespan. Brain Behav. Immun. 27, 8-12. doi: 10.1016/J.BBI.2012.06.014

Ferrone, C. K., Blydt-Hansen, M., and Rauh, M. J. (2020). Age-associated TET2 mutations: common drivers of myeloid dysfunction, cancer and cardiovascular disease. Int. J. Mol. Sci. 21:626. doi: 10.3390/ijms21020626

Fuster, J. J., MacLauchlan, S., Zuriaga, M. A., Polackal, M. N., Ostriker, A. C., Chakraborty, R., et al. (2017). Clonal hematopoiesis associated with TET2 deficiency accelerates atherosclerosis development in mice. Science 355, 842847. doi: $10.1126 /$ science.aag 1381 
Gilmore, T. D. (2006). Introduction to NF-кB: players, pathways, perspectives. Oncogene 25, 6680-6684. doi: 10.1038/sj.onc.1209954

Goldberg, A. D., Allis, C. D., and Bernstein, E. (2007). Epigenetics: a landscape takes shape. Cell 128, 635-638. doi: 10.1016/J.CELL.2007.02.006

Gong, F., Guo, Y., Niu, Y., Jin, J., Zhang, X., Shi, X., et al. (2016). Epigenetic silencing of TET2 and TET3 induces an EMT-like process in melanoma. Oncotarget 8:1.

Goodfellow, F. T., Tesla, B., Simchick, G., Zhao, Q., Hodge, T., Brindley, M. A., et al. (2016). Zika virus induced mortality and microcephaly in chicken embryos. Stem Cells Dev. 25, 1691-1697. doi: 10.1089/scd.2016.0231

Gouin, J.-P., Glaser, R., Malarkey, W. B., Beversdorf, D., and Kiecolt-Glaser, J. K. (2012). Childhood abuse and inflammatory responses to daily stressors. Ann. Behav. Med. 44, 287-292. doi: 10.1007/s12160-012-9386-1

Hales, C., and Barker, D. (1992). Type 2 (non-insulin-dependent) diabetes mellitus: the thrifty phenotype hypothesis. Diabetologia $35,595-601$.

Haller, S., Ametamey, S. M., Schibli, R., and Müller, C. (2015). Investigation of the chick embryo as a potential alternative to the mouse for evaluation of radiopharmaceuticals. Nucl. Med. Biol. 42, 226-233. doi: 10.1016/J. NUCMEDBIO.2014.10.010

Hassan, N., Ali, A., Withycombe, C., Ahluwalia, M., Al-Nasseri, R. H., Tonks, A., et al. (2018). TET-2 up-regulation is associated with the anti-inflammatory action of Vicenin-2. Cytokine 108, 37-42. doi: 10.1016/J.CYTO.2018. 03.016

Hoffmann, A., Zimmermann, C. A., and Spengler, D. (2015). Molecular epigenetic switches in neurodevelopment in health and disease. Front. Behav. Neurosci. 9:120. doi: 10.3389/fnbeh.2015.00120

Horowitz, M. (2017). Heat acclimation-mediated cross-tolerance: origins in within-life epigenetics? Front. Physiol. 8:548. doi: 10.3389/fphys.2017.00548

Horowitz, M., Umschweif, G., Yacobi, A., and Shohami, E. (2015). Molecular programs induced by heat acclimation confer neuroprotection against TBI and hypoxic insults via cross-tolerance mechanisms. Front. Neurosci. 9:256. doi: 10.3389/fnins.2015.00256

Hossain, M. A., Li, Z.-G., Hoque, T. S., Burritt, D. J., Fujita, M., and Munné-Bosch, S. (2018). Heat or cold priming-induced cross-tolerance to abiotic stresses in plants: key regulators and possible mechanisms. Protoplasma 255, 399-412. doi: 10.1007/s00709-017-1150-8

Hueston, C. M., and Deak, T. (2014). The inflamed axis: the interaction between stress, hormones, and the expression of inflammatory-related genes within key structures comprising the hypothalamic-pituitary-adrenal axis. Physiol. Behav. 124, 77-91. doi: 10.1016/j.physbeh.2013.10.035

Itoh, H., Kadomatsu, T., Tanoue, H., Yugami, M., Miyata, K., Endo, M., et al. (2018). TET2-dependent IL-6 induction mediated by the tumor microenvironment promotes tumor metastasis in osteosarcoma. Oncogene 37, 2903-2920. doi: 10.1038/s41388-018-0160-0

Jais, A., and Brüning, J. C. (2017). Hypothalamic inflammation in obesity and metabolic disease. J. Clin. Invest. 127, 24-32. doi: 10.1172/JCI88878

Kadhim, H. J., Kang, S. W., and Kuenzel, W. J. (2019). Differential and temporal expression of corticotropin releasing hormone and its receptors in the nucleus of the hippocampal commissure and paraventricular nucleus during the stress response in chickens (Gallus gallus). Brain Res. 1714, 1-7. doi: 10.1016/j. brainres.2019.02.018

Katz, A., and Meiri, N. (2006). Brain-derived neurotrophic factor is critically involved in thermal-experience-dependent developmental plasticity. J. Neurosci. 26, 3899-3907. doi: 10.1523/JNEUROSCI.0371-06.2006

Kisliouk, T., Cramer, T., and Meiri, N. (2017). Methyl CpG level at distal part of heat-shock protein promoter HSP70 exhibits epigenetic memory for heat stress by modulating recruitment of POU2F1-associated nucleosome-remodeling deacetylase (NuRD) complex. J. Neurochem. 141, 358-372. doi: 10.1111/jnc. 14014

Kisliouk, T., and Meiri, N. (2009). A critical role for dynamic changes in histone $\mathrm{H} 3$ methylation at the Bdnf promoter during postnatal thermotolerance acquisition. Eur. J. Neurosci. 30, 1909-1922. doi: 10.1111/j.1460-9568.2009. 06957.x

Kisliouk, T., Ziv, M., and Meiri, N. (2009). Epigenetic control of translation regulation: alterations in histone $\mathrm{H} 3$ lysine 9 post-translation modifications are correlated with the expression of the translation initiation factor 2B (Eif2b5) during thermal control establishment. Dev. Neurobiol. 70, 100-113. doi: 10. 1002/dneu.20763
Klengel, T., and Binder, E. B. (2015). miller. Neuron 86, 1343-1357. doi: 10.1016/j. neuron.2015.05.036

Klett, H., Rodriguez-Fernandez, M., Dineen, S., Leon, L. R., Timmer, J., and Doyle, F. J. (2015). Modeling the inflammatory response in the hypothalamus ensuing heat stroke: iterative cycle of model calibration, identifiability analysis, experimental design and data collection. Math. Biosci. 260, 35-46. doi: 10.1016/ j.mbs.2014.07.011

Kohli, R. M., and Zhang, Y. (2013). TET enzymes, TDG and the dynamics of DNA demethylation. Nature 502, 472-479. doi: 10.1038/nature12750

Kumar, A., Chen, S. H., Kadiiska, M. B., Hong, J. S., Zielonka, J., Kalyanaraman, B., et al. (2014). Inducible nitric oxide synthase is key to peroxynitrite-mediated, LPS-induced protein radical formation in murine microglial BV2 cells. Free Radic. Biol. Med. 73, 51-59. doi: 10.1016/j.freeradbiomed.2014.04.014

Labonté, B., Suderman, M., Maussion, G., Navaro, L., Yerko, V., Mahar, I., et al. (2012). Genome-wide epigenetic regulation by early-life trauma. Arch. Gen. Psychiatry 69, 722-731. doi: 10.1001/archgenpsychiatry.2011.2287

Ligthart, S., Marzi, C., Aslibekyan, S., Mendelson, M. M., Conneely, K. N., Tanaka, T., et al. (2016). DNA methylation signatures of chronic low-grade inflammation are associated with complex diseases. Genome Biol. 17:255. doi: 10.1186/s13059-016-1119-5

Liu, J. J., Buisman-Pijlman, F., and Hutchinson, M. R. (2014). Toll-like receptor 4: innate immune regulator of neuroimmune and neuroendocrine interactions in stress and major depressive disorder. Front. Neurosci. 8:309. doi: 10.3389/fnins. 2014.00309

Liu, P., Wang, H., Li, X., Chao, T., Teav, T., Christen, S., et al. (2017). A -ketoglutarate orchestrates macrophage activation through metabolic and epigenetic reprogramming. Nat. Immunol. 18, 985-994. doi: 10.1038/ni.3796

Loyau, T., Bedrani, L., Berri, C., Métayer-Coustard, S., Praud, C., Coustham, V., et al. (2014). Cyclic variations in incubation conditions induce adaptive responses to later heat exposure in chickens: a review. Animal 9, 76-85. doi: $10.1017 /$ S1751731114001931

Loyau, T., Hennequet-Antier, C., Coustham, V., Berri, C., Leduc, M., Crochet, S., et al. (2016). Thermal manipulation of the chicken embryo triggers differential gene expression in response to a later heat challenge. BMC Genomics 17:329. doi: 10.1186/s12864-016-2661-y

Lupien, S. J., McEwen, B. S., Gunnar, M. R., and Heim, C. (2009). Effects of stress throughout the lifespan on the brain, behaviour and cognition. Nat. Rev. Neurosci. 10, 434-445. doi: 10.1038/nrn2639

Macrez, R., Ali, C., Toutirais, O., Le Mauff, B., Defer, G., Dirnagl, U., et al. (2011). Stroke and the immune system: from pathophysiology to new therapeutic strategies. Lancet Neurol. 10, 471-480. doi: 10.1016/S1474-4422(11)7 0066-7

Martinez, F. O., and Gordon, S. (2014). The M1 and M2 paradigm of macrophage activation: time for reassessment. F1000Prime Rep. 6:13. doi: 10.12703/P6-13

Matsuzaki, K., Katakura, M., Sugimoto, N., Hara, T., Hashimoto, M., and Shido, O. (2017). Neural progenitor cell proliferation in the hypothalamus is involved in acquired heat tolerance in long-term heat-acclimated rats. PLoS One 12:e0178787. doi: 10.1371/journal.pone.0178787

Matt, S. M., Zimmerman, J. D., Lawson, M. A., Bustamante, A. C., Uddin, M., and Johnson, R. W. (2018). Inhibition of DNA methylation with zebularine alters lipopolysaccharide-induced sickness behavior and neuroinflammation in mice. Front. Neurosci. 12:636. doi: 10.3389/fnins.2018.00636

Meaney, M. J., and Szyf, M. (2005). Environmental programming of stress responses through DNA methylation: life at the interface between a dynamic environment and a fixed genome. Dialogues Clin. Neurosci. 7, 103-123.

Melamed, P., Yosefzon, Y., David, C., Tsukerman, A., and Pnueli, L. (2018). Tet enzymes, variants, and differential effects on function. Front. Cell Dev. Biol. 6:22. doi: $10.3389 /$ fcell.2018.00022

Miller, G. E., Chen, E., Fok, A. K., Walker, H., Lim, A., Nicholls, E. F., et al. (2009). Low early-life social class leaves a biological residue manifested by decreased glucocorticoid and increased proinflammatory signaling. PNAS 106, 14716-14721. doi: 10.1073/pnas.0902971106

Morrison, S. F. (2016). Central control of body temperature. F1000Research 5:F1000FacultyRev-880. doi: 10.12688/f1000research.7958.1

Nassar, M., Halle, I., Plagemann, A., and Tzschentke, B. (2015). Detection of long-term influence of prenatal temperature stimulation on hypothalamic type-II iodothyronine deiodinase in juvenile female broiler chickens using a novel immunohistochemical amplification protocol. Comp. Biochem. Physiol. 
Part A Mol. Integr. Physiol. 179, 120-124. doi: 10.1016/J.CBPA.2014. 09.038

Niu, Y., DesMarais, T. L., Tong, Z., Yao, Y., and Costa, M. (2015). Oxidative stress alters global histone modification and DNA methylation. Free Radic. Biol. Med. 82, 22-28. doi: 10.1016/j.freeradbiomed.2015.01.028

Pace, T. W. W., Mletzko, T. C., Alagbe, O., Musselman, D. L., Nemeroff, C. B., Miller, A. H., et al. (2006). Increased stress-induced inflammatory responses in male patients with major depression and increased early life stress. Am. J. Psychiatry 163, 1630-1633. doi: 10.1176/ajp.2006.163.9.1630

Piestun, Y., Halevy, O., and Yahav, S. (2009). Thermal manipulations of broiler embryos-The effect on thermoregulation and development during embryogenesis1. Poult. Sci. 88, 2677-2688.

Piestun, Y., Shinder, D., Ruzal, M., Halevy, O., Brake, J., and Yahav, S. (2008). Thermal manipulations during broiler embryogenesis: effect on the acquisition of thermotolerance1. Poult. Sci. 87, 1516-1525.

Piestun, Y., Yahav, S., and Halevy, O. (2015). Thermal manipulation during embryogenesis affects myoblast proliferation and skeletal muscle growth in meat-type chickens. Poult. Sci. 94, 2528-2536. doi: 10.3382/ps/pev245

Pollak, A., Merin, G., Horowitz, M., Shochina, M., Gilon, D., and Hasin, Y. (2017). Heat acclimatization protects the left ventricle from increased diastolic chamber stiffness immediately after coronary artery bypass surgery: a lesson from 30 years of studies on heat acclimation mediated cross tolerance. Front. Physiol. 8:1022. doi: 10.3389/fphys.2017.01022

Riccardi, C. (2015). GILZ as a mediator of the anti-inflammatory effects of glucocorticoids. Front. Endocrinol. 6:170. doi: 10.3389/fendo.2015.00170

Roesslein, M., Froehlich, C., Jans, F., Piegeler, T., Goebel, U., Loop, T., et al. (2015). Hypothalamic inflammation in the control of metabolic function. Comp. Biochem. Physiol. A Mol. Integr. Physiol. 9, 475-483. doi: 10.3389/fnins. 2014.00447

Ropelle, E. R., Flores, M. B., Cintra, D. E., Rocha, G. Z., Pauli, J. R., Morari, J., et al. (2010). IL-6 and IL-10 anti-inflammatory activity links exercise to hypothalamic insulin and leptin sensitivity through IKK $\beta$ and ER stress inhibition. PLoS Biol. 8:e1000465. doi: 10.1371/journal.pbio.1000465

Ruben, O., Christopher, A. M., and Jean, H. G. (2015). Microglial M1/M2 polarization and metabolic states. Br. J. Pharmacol. 173, 649-665. doi: 10.1111/ bph.13139

Sengupta, T., Jaryal, A. K., and Mallick, H. N. (2016). Effects of NMDA and nonNMDA ionotropic glutamate receptors in the medial preoptic area on body temperature in awake rats. J. Therm. Biol. 61, 1-7. doi: 10.1016/j.jtherbio.2016. 07.020

Shein, N. A., Grigoriadis, N., Alexandrovich, A. G., Simeonidou, C., Spandou, E., Tsenter, J., et al. (2008). Differential neuroprotective properties of endogenous and exogenous erythropoietin in a mouse model of traumatic brain injury. J. Neurotrauma 25, 112-123. doi: 10.1089/neu.2007.0358

Shi, D. Q., Ali, I., Tang, J., and Yang, W. C. (2017). New insights into 5hmC DNA modification: generation, distribution and function. Front. Genet. 8:100. doi: 10.3389/fgene.2017.00100

Shonkoff, J. P., Boyce, W. T., and McEwen, B. S. (2009). Neuroscience, molecular biology, and the childhood roots of health disparities: building a new framework for health promotion and disease prevention. JAMA J. Am. Med. Assoc. 301, 2252-2259. doi: 10.1001/jama.2009.754

Skiles, W. M., Kester, A., Pryor, J. H., Westhusin, M. E., Golding, M. C., and Long, C. R. (2018). Oxygen-induced alterations in the expression of chromatin modifying enzymes and the transcriptional regulation of imprinted genes. Gene Expr. Patterns 28, 1-11. doi: 10.1016/j.gep.2018.01.001

Smith, S. M., and Vale, W. W. (2006). The role of the hypothalamic-pituitaryadrenal axis in neuroendocrine responses to stress. Dialogues Clin. Neurosci. 8, 383-395.

Star, L., Juul-Madsen, H. R., Decuypere, E., Nieuwland, M. G. B., de Vries Reilingh, G., van den Brand, H., et al. (2009). Effect of early life thermal conditioning and immune challenge on thermotolerance and humoral immune competence in adult laying hens. Poult. Sci. 88, 2253-2261. doi: 10.3382/ps.2008-00373

Tai, P.-A., Chang, C.-K., Niu, K.-C., Lin, M.-T., Chiu, W.-T., and Lin, J.-W. (2010). Attenuation of heat-induced hypothalamic ischemia, inflammation, and damage by hyperbaric oxygen in rats. J. Neurotrauma doi: 10.1089/neu.2010. 1323 [Epub ahead of print].

Tan, J., Liu, S., Guo, Y., Applegate, T. J., and Eicher, S. D. (2014). Dietary 1-arginine supplementation attenuates lipopolysaccharide-induced inflammatory response in broiler chickens. Br. J. Nutr. 111, 1394-1404. doi: $10.1017 /$ S0007114513003863

Tang, Y., and Le, W. (2016). Differential roles of M1 and M2 microglia in neurodegenerative diseases. Mol. Neurobiol. 53, 1181-1194. doi: 10.1007/ s12035-014-9070-5

Taylor, S. E. (2010). Mechanisms linking early life stress to adult health outcomes. Proc. Natl. Acad. Sci. U.S.A. 107, 8507-8512. doi: 10.1073/pnas.1003890107

Tona, K., Onagbesan, O., Bruggeman, V., Collin, A., Berri, C., Duclos, M. J., et al. (2008). Effects of heat conditioning at d 16 to 18 of incubation or during early broiler rearing on embryo physiology, post-hatch growth performance and heat tolerance. Eur. Poult. Sci. 72, 75-83.

Turecki, G., and Meaney, M. J. (2016). Effects of the social environment and stress on glucocorticoid receptor gene methylation: a systematic review. Biol. Psychiatry 79, 87-96. doi: 10.1016/J.BIOPSYCH.2014.11.022

Tzschentke, B. (2007). Attainment of thermoregulation as affected by environmental factors. Poult. Sci. 86, 1025-1036. doi: 10.1093/ps/86.5.1025

Tzschentke, B., and Halle, I. (2009). Influence of temperature stimulation during the last 4 days of incubation on secondary sex ratio and later performance in male and female broiler chicks. Br. Poult. Sci. 50, 634-640. doi: 10.1080/ 00071660903186570

Umschweif, G., Shabashov, D., Alexandrovich, A. G., Trembovler, V., Horowitz, M., and Shohami, E. (2014). Neuroprotection after traumatic brain injury in heat-acclimated mice involves induced neurogenesis and activation of angiotensin receptor type 2 signaling. J. Cereb. Blood Flow Metab. 34, 13811390. doi: 10.1038/jcbfm.2014.93

Umschwief, G., Shein, N. A., Alexandrovich, A. G., Trembovler, V., Horowitz, M., and Shohami, E. (2010). Heat acclimation provides sustained improvement in functional recovery and attenuates apoptosis after traumatic brain injury. J. Cereb. Blood Flow Metab. 30, 616-627. doi: 10.1038/jcbfm. 2009.234

Valdearcos, M., Xu, A. W., and Koliwad, S. K. (2015). Hypothalamic inflammation in the control of metabolic function. Annu. Rev. Physiol. 77, 131-160. doi: 10.1146/annurev-physiol-021014-071656

Vento-Tormo, R., Álvarez-Errico, D., Garcia-Gomez, A., Hernández-Rodríguez, J., Buján, S., Basagaña, M., et al. (2017). DNA demethylation of inflammasomeassociated genes is enhanced in patients with cryopyrin-associated periodic syndromes. J. Allergy Clin. Immunol. 139, 202.e6-211.e6. doi: 10.1016/J.JACI. 2016.05.016

Vernardis, S. I., Terzoudis, K., Panoskaltsis, N., and Mantalaris, A. (2017). Human embryonic and induced pluripotent stem cells maintain phenotype but alter their metabolism after exposure to ROCK inhibitor. Sci. Rep. 7:42138. doi: $10.1038 /$ srep 42138

Wallner, S., Schröder, C., Leitão, E., Berulava, T., Haak, C., Beißer, D., et al. (2016). Epigenetic dynamics of monocyte-to-macrophage differentiation. Epigenet. Chromatin 9:33. doi: 10.1186/s13072-016-0079-Z

Wang, X., Feng, Z., Li, Q., Yi, B., and Xu, Q. (2018). DNA methylcytosine dioxygenase ten-eleven translocation 2 enhances lipopolysaccharide-induced cytokine expression in human dental pulp cells by regulating MyD88 hydroxymethylation. Cell Tissue Res. 373, 477-485. doi: 10.1007/s00441-0182826-x

Wang, X., Stridh, L., Li, W., Dean, J., Elmgren, A., Gan, L., et al. (2009). Lipopolysaccharide sensitizes neonatal hypoxic-ischemic brain injury in a MyD88-dependent manner. J. Immunol. 183, 7471-7477. doi: 10.4049/ JIMMUNOL.0900762

Wu, B., Liu, J., Zhao, R., Li, Y., Peer, J., Braun, A. L., et al. (2018). Glutaminase 1 regulates the release of extracellular vesicles during neuroinflammation through key metabolic intermediate alpha-ketoglutarate. J. Neuroinflammation 15:79. doi: 10.1186/s12974-018-1120-x

$\mathrm{Wu}, \mathrm{X}$., and Zhang, Y. (2017). TET-mediated active DNA demethylation: mechanism, function and beyond. Nat. Rev. Genet. 18, 517-534. doi: 10.1038/ nrg.2017.33

Xiang, W., Chao, Z.-Y., and Feng, D.-Y. (2015). Role of Toll-like receptor/MYD88 signaling in neurodegenerative diseases. Rev. Neurosci. 26, 407-414. doi: 10. 1515/revneuro-2014-0067

Yacobi, A., Stern Bach, Y., and Horowitz, M. (2014). The protective effect of heat acclimation from hypoxic damage in the brain involves changes in the expression of glutamate receptors. Temperature 1, 57-65. doi: 10.4161/temp. 29719 
Yang, C., Li, Z., Kang, W., Tian, Y., Yan, Y., and Chen, W. (2016). TET1 and TET3 are essential in induction of Th2-type immunity partly through regulation of IL4/13A expression in zebrafish model. Gene 591, 201-208. doi: 10.1016/J.GENE. 2016.07.025

Yossifoff, M., Kisliouk, T., and Meiri, N. (2008). Dynamic changes in DNA methylation during thermal control establishment affect CREB binding to the brain-derived neurotrophic factor promoter. Eur. J. Neurosci. 28, 2267-2277. doi: 10.1111/j.1460-9568.2008.06532.x

Zhang, Q., Zhao, K., Shen, Q., Han, Y., Gu, Y., Li, X., et al. (2015). Tet2 is required to resolve inflammation by recruiting Hdac2 to specifically repress IL-6. Nature 525, 389-393. doi: 10.1038/nature15252
Conflict of Interest: The authors declare that the research was conducted in the absence of any commercial or financial relationships that could be construed as a potential conflict of interest.

Copyright ( 2020 Rosenberg, Kisliouk, Cramer, Shinder, Druyan and Meiri. This is an open-access article distributed under the terms of the Creative Commons Attribution License (CC BY). The use, distribution or reproduction in other forums is permitted, provided the original author(s) and the copyright owner(s) are credited and that the original publication in this journal is cited, in accordance with accepted academic practice. No use, distribution or reproduction is permitted which does not comply with these terms. 